\title{
On the Regularity of Weak Contact $p$-Harmonic Maps
}

\author{
Sorin Dragomir ${ }^{1}$ and Robert Petit ${ }^{2}$ \\ ${ }^{1}$ Università degli Studi della Basilicata, Dipartimento di Matematica, Informatica ed Economia, Via Dell'Ateneo Lucano 10, Contrada \\ Macchia Romana, 85100 Potenza, Italy \\ ${ }^{2}$ Laboratoire de Mathématiques Jean Leray, UMR 6629 CNRS, Université de Nantes, 2, rue de la Houssinière, BP 92208, \\ 44322 Nantes, France
}

Correspondence should be addressed to Sorin Dragomir; sorin.dragomir@unibas.it

Received 11 September 2012; Accepted 26 January 2013

Academic Editor: Arcadii Grinshpan

Copyright (C) 2013 S. Dragomir and R. Petit. This is an open access article distributed under the Creative Commons Attribution License, which permits unrestricted use, distribution, and reproduction in any medium, provided the original work is properly cited.

We prove Caccioppoli type estimates and consequently establish local Hölder continuity for a class of weak contact $(2 n+2)$ harmonic maps from the Heisenberg group $\mathbb{H}_{n}$ into the sphere $S^{2 m-1}$.

\section{Introduction}

The study of pseudoharmonic maps was started by Barletta et al. [1] (cf. also $[2,3]$ for successive investigations) as a generalization of the theory of harmonic maps among Riemannian manifolds (cf., e.g., [4]) and by identifying the results of Jost and $\mathrm{Xu}$ [5], Zhou [6], Hajłasz and Strzelecki [7], and Wang [8] as local aspects of the theory of pseudoharmonic maps from a strictly pseudoconvex CR manifold into a Riemannian manifold (cf. also [9, pages 225-226]).

A similar class of maps, yet with values in another CR manifold, was studied in [10]. These are critical points of the functional

$$
E(\phi)=\frac{1}{2} \int_{M} Q(\phi) d v, \quad \phi \in C^{\infty}(M, N),
$$

where $M$ is a compact strictly pseudoconvex CR manifold of CR dimension $n, Q(\phi)=\left\|(d \phi)_{H, H^{\prime}}\right\|^{2}, d v=\theta \wedge(d \theta)^{n}$, and $\theta$ is a contact form on $M$. Also $N$ is a contact Riemannian manifold and in particular an almost CR manifold (of CR codimension 1).

A moment's thought reveals the augmented difficulties such a theory may present. For instance, if $M$ and $N$ are two strictly pseudoconvex CR manifolds endowed, respectively, with contact forms $\theta$ and $\eta$, then the pseudohermitian analog of the notion of a harmonic morphism (among Riemannian manifolds) is quite obvious: one may consider continuous maps $\phi: M \rightarrow N$ such that the pullback $v \circ \phi$ of any local solution $v: U^{\prime} \subseteq N \rightarrow \mathbb{R}$ to $\Delta_{b}^{N} v=0$ in $V$ satisfies $\Delta_{b}(v \circ \phi)=0$ in $U=\phi^{-1}\left(U^{\prime}\right)$ in distribution sense. Here $\Delta_{b}$ and $\Delta_{b}^{N}$ are the sublaplacians of $(M, \theta)$ and $(N, \eta)$, respectively. Unlike the situation in [2] (where the target manifold $N$ is Riemannian and $\phi$ pulls back local harmonic functions on $N$ to distribution solutions of $\Delta_{b} u=0$ ) such $\phi$ is not necessarily smooth (since it is unknown whether local coordinate systems $\left(U^{\prime}, x^{\prime i}\right)$ on $N$ such that $\Delta_{b}^{N} x^{\prime i}=0$ in $U^{\prime}$ might be produced). To give another example, should one look for a pseudohermitian analog to the Fluglede-Ishihara theorem (cf. [3] when $M$ is CR and $N$ is Riemannian), one would face the lack of an Ishihara type lemma (cf. [11]) as it is unknown whether $\Delta_{b}^{N} v=0$ admits local solutions whose (horizontal) gradient and hessian have prescribed values at a point. Moreover, what would be the appropriate notion of a hessian (cf. [12] for a possible choice)?

A third example, discussed at some length in this paper, is that of the "degeneracy" of the Euler-Lagrange equations

$$
\begin{aligned}
& {\left[\left(\varphi^{2}\right)_{j}^{i} \circ \phi\right]} \\
& \quad \times\left\{\operatorname{div}\left[Q(\phi)^{(p-2) / 2} \nabla^{H} \phi^{j}\right]\right.
\end{aligned}
$$




$$
\begin{array}{r}
\left.+Q(\phi)^{(p-2) / 2} \sum_{a=1}^{2 n}\left(\Gamma_{k \ell}^{\prime j} \circ \phi\right) X_{a}\left(\phi^{k}\right) X_{a}\left(\phi^{\ell}\right)\right\}=0 \\
1 \leq i \leq 2 m-1
\end{array}
$$

associated to the variational principle

$$
\delta \int Q(\phi)^{p / 2} d v=0
$$

when $N$ is a Sasakian manifold. Indeed the $(2 m-1) \times(2 m-1)$ matrix $\left(\varphi^{2}\right)_{j}^{i}=-\delta_{j}^{i}+\xi^{i} \eta_{j}$ has but rank $2 m-2$ at each point (a well-known phenomenon in contact Riemannian geometry, cf., e.g., [13]. See also [14]). Consequently, in general one may not expect regularity of weak solutions to (2). For instance, if $N=\mathbb{W}_{m-1}$ is the Heisenberg group and $\phi=\left(\phi^{\prime}, \phi^{2 m-1}\right): U \subseteq$ $M \rightarrow \mathbb{H}_{m-1}$ is a solution to (2), then $\phi^{\prime}: U \rightarrow \mathbb{R}^{2 m-2}$ is subject to

$$
\sum_{a=1}^{2 n} X_{a}^{*}\left(\left|X \phi^{\prime}\right|^{p-2} X_{a}\left(\phi^{i}\right)\right)=0, \quad 1 \leq i \leq 2 m-2
$$

yet $\phi^{2 m-1}$ is an arbitrary function (cf. Section 3). For the more appealing case, where $M=\mathbb{U}_{n}$ is the Heisenberg group and $N=S^{2 m-1}$ is the sphere, (2) may be written as

$$
X^{*} \cdot V_{A}=Q(\phi)^{p / 2} \phi_{A}, \quad 1 \leq A \leq 2 m,
$$

(cf. Proposition 15) which is indeed the form assumed by the Euler-Lagrange equations in [7], yet unlike the situation there $X^{*} \cdot E_{A, B} \neq 0$ in general (cf. Proposition 16 for the notations). Although $X^{*} \cdot E_{A, B}$ has a quite explicit form (yielding-for a class of weak solutions $\phi: \mathbb{H}_{n} \rightarrow S^{2 m-1}$ which are close to being horizontal maps-simple estimates on $X^{*} \cdot E_{A, B}$ ), only a weaker form of the duality inequality lemma in [7] may be proved (cf. Lemma 17) leading nevertheless (together with a hole filling argument) to Caccioppoli type estimates

$$
\int_{B_{X}(x, r)}|X \phi|^{2 n+2} d v \leq C r^{\gamma}
$$

for some $C>0$ and $0<\gamma<1$, which are known (cf., e.g., [7] for a very general argument based on work in [15]) to imply the local Hölder continuity of the given weak solution.

The paper is organized as follows. In Section 2 we recall a few conventions and basic results obtained in [10]. Sections 3 and 4 are devoted to the study of the local properties of weak contact $p$-harmonic maps. We show that weak contact $(2 n+2)$-maps $\phi: U \subset \mathbb{H}_{n} \rightarrow S^{2 m-1}$ are locally Hölder continuous (cf. Corollary 21) provided they are close to being horizontal maps; that is, the assumptions (96) are satisfied. The relevance of the number $p=2 n+2$ stems from the facts that $\int_{M}\left\|(d \phi)_{H, H^{\prime}}\right\|^{2 n+2} d v$ is a CR invariant and $2 n+2$ is the homogeneous dimension of $\mathbb{H}_{n}$. The authors believe that subelliptic theory should play within CR geometry, as a branch of complex analysis in several complex variables, the strong role played by elliptic theory in Riemannian geometry, and the present paper is a step in this direction.

\section{Basic Conventions and Results}

For all notions of CR and pseudohermitian geometry we adopt the conventions and notations in the monograph [9]. For the approach to contact structures within Riemannian geometry we rely on the presentation in Blair [13], (cf. also Tanno [16]). Given a real $(2 n+1)$-dimensional $C^{\infty}$ differentiable manifold $M$, an almost $C R$ structure is a complex subbundle $T_{1,0}(M) \subset T(M) \otimes \mathbb{C}$ of the complexified tangent bundle, of complex rank $n$, such that $T_{1,0}(M)_{x} \cap$ $T_{0,1}(M)_{x}=(0)$ for any $x \in M$. Here $T_{0,1}(M)=\overline{T_{1,0}(M)}$ and overbars indicate complex conjugates. The integer $n$ is the $C R$ dimension of the almost CR manifold $\left(M, T_{1,0}(M)\right)$. Almost CR structures are a bundle theoretic recast of the tangential Cauchy-Riemann operator $\bar{\partial}_{b}: C^{\infty}(M, \mathbb{C}) \rightarrow C^{\infty}\left(T_{0,1}(M)^{*}\right)$ given by $\left(\bar{\partial}_{b} f\right) \bar{Z}=\bar{Z}(f)$ for any $f \in C^{\infty}(M, \mathbb{C})$ and any $Z \in$ $T_{1,0}(M)$. An almost CR structure is (formally or Frobenius) integrable if $[Z, W] \in C^{\infty}\left(U, T_{1,0}(M)\right)$ for any $Z, W \in$ $C^{\infty}\left(U, T_{1,0}(M)\right)$ and any open set $U \subset M$. The tangential C-R operator may be extended to arbitrary $(0, q)$-forms on $M$ and the resulting pseudocomplex $\bar{\partial}_{b}: \Omega^{0, q}(M) \rightarrow \Omega^{0, q+1}(M)$, $q \geq 0$, is a complex (i.e., $\bar{\partial}_{b}^{2}=0$ ) if and only if the given almost CR structure is integrable (cf. [9]). Integrable almost CR structures are commonly referred to as CR structures and appear mainly on real hypersurfaces of complex manifolds, as induced by the complex structure of the ambient space; that is, for any complex manifold $V$ and any real hypersurface $M \subset V$

$$
T_{1,0}(M)_{x}=\left[T_{x}(M) \otimes_{\mathbb{R}} \mathbb{C}\right] \cap T^{1,0}(V)_{x}, \quad x \in M,
$$

is a CR structure on $M$. Here $T^{1,0}(V) \rightarrow V$ is the holomorphic tangent bundle over $V$ (locally the span of $\left\{\partial / \partial z^{j}: 1 \leq j \leq N\right\}$ for any local system of complex coordinates $\left(z^{j}\right)$ on $V$ ). Also $N$ is the complex dimension of $V$, and then the CR dimension of $M$ is $n=N-1$. Integrability of (7) follows from the Nijenhuis integrability of the complex structure on $V$. A solution $f$ to $\bar{\partial}_{b} f=0$ (the tangential $C-R$ equations) is a $C R$ function on $M$ and, in the context of real hypersurfaces carrying the induced CR structure (7), CR functions appear as traces on $M$ of holomorphic functions defined on a neighborhood of $M$ in $V$. Hence to say that the CR structure is given by (7) is to say that the tangential C-R equations are induced by the ordinary Cauchy-Riemann system on $V$. CR functions which are not traces of holomorphic functions may exist (cf., e.g., [17]). CR structures which are not given by (7), and for which there is not any embedding of $M$ into some complex manifold $V$ yielding (7), do exist as well (cf. again [17, page 172]). An array of geometric objects, such as pseudohermitian structures, the Levi form (cf. $[9,18]$ ) and successively (in the nondegenerate case) contact structures, the Tanaka-Webster connection (cf. $[18,19]$ ), the sublaplacian $\Delta_{b}$ and the Fefferman metric (cf. $[9,20])$, springs from the given CR structure very much the way the complex structure determines the metric structure (up to a conformal invariant) on a Riemann surface and are thought of as geometric tools whose use will ultimately shed light on the properties of solutions, local and global, to the 
tangential C-R equations. Integrability of $T_{1,0}(M)$ appears as a built-in ingredient of objects such as the Tanaka-Webster connection or the Fefferman metric, yet it is believed to lack the geometric meaning of involutivity of real smooth distributions on manifolds (cf., e.g., [21, page 16]). On the other hand nonintegrable examples of almost CR structures occur frequently, either on real hypersurfaces of almost complex manifolds or on contact Riemannian manifolds (cf. $[13,16])$. A remedy was indicated by Tanno [16], showing that the wealth of additional structure $(\varphi, \xi, \eta, g)$ on a given contact Riemannian manifold $N$ compensates for the lack of integrability of $T_{1,0}(N)=\{X-i \varphi X: X \in \operatorname{Ker}(\eta)\}$ and specifically providing a generalization of the Tanaka-Webster connection to the nonintegrable context.

Given a CR manifold $\left(M, T_{1,0}(M)\right)$, let $H=\operatorname{Re}\left\{T_{1,0}(M) \oplus\right.$ $\left.T_{0,1}(M)\right\}$ be the Levi, or maximally complex, distribution and $J(Z+\bar{Z})=i(Z-\bar{Z}), Z \in T_{1,0}(M)$, its complex structure. Let $H_{x}^{\perp}=\left\{\omega \in T_{x}^{*}(M): \operatorname{Ker}(\omega) \supseteq H_{x}\right\}, x \in M$, be the conormal bundle associated to $H$, a real line bundle over $M$. Since $M$ is assumed to be connected and orientable, the conormal bundle $H^{\perp} \rightarrow M$ is trivial. A globally defined nowhere zero section $\theta \in \Gamma^{\infty}\left(H^{\perp}\right)$ is a pseudohermitian structure on $M$. For each pseudohermitian structure $\theta$ on $M$ the Levi form is

$$
G_{\theta}(X, Y)=(d \theta)(X, J Y), \quad X, Y \in H .
$$

Two pseudohermitian structures $\theta, \hat{\theta} \in \Gamma^{\infty}\left(H^{\perp}\right)$ are related by $\widehat{\theta}=\lambda \theta$ for some $C^{\infty}$ function $\lambda: M \rightarrow \mathbb{R} \backslash\{0\}$. If this is the case, then $G_{\hat{\theta}}=\lambda G_{\theta}$. A CR manifold $M$ is nondegenerate (resp., strictly pseudoconvex) if $G_{\theta}$ is nondegenerate (resp., positive definite) for some $\theta$. If $M$ is a nondegenerate CR manifold, of CR dimension $n$, then each pseudohermitian structure $\theta$ is a contact form; that is, $\theta \wedge(d \theta)^{n}$ is a volume form on $M$. If $M$ is nondegenerate and $\theta$ is a contact form on $M$, there is a unique globally defined, nowhere zero, tangent vector field $T \in \mathfrak{X}^{\infty}(M)$ (the Reeb vector field of $(M, \theta)$ ) such that $\theta(T)=1$ and $(d \theta)(T, \cdot)=0$. The Webster metric is the semi-Riemannian metric $g_{\theta}$ on $M$ given by

$$
g_{\theta}(X, Y)=G_{\theta}(X, Y), \quad g_{\theta}(X, T)=0, \quad g_{\theta}(T, T)=1,
$$

for any $X, Y \in H$. If $M$ is strictly pseudoconvex and $\theta$ is chosen such that $G_{\theta}$ is positive definite, then $g_{\theta}$ is a Riemannian metric on $M$.

Let $N$ be a $(2 m-1)$-dimensional $C^{\infty}$ manifold $(m \geq 2)$. An almost contact structure on $N$ is a synthetic object $(\phi, \xi, \eta)$ consisting of a $(1,1)$-tensor field $\varphi$, a vector field $\xi \in \mathfrak{X}^{\infty}(N)$, and a 1-form $\eta \in \Omega^{1}(N)$ such that

$$
\begin{gathered}
\varphi_{k}^{i} \varphi_{j}^{k}=-\delta_{j}^{i}+\eta_{j} \xi^{i}, \quad \eta_{i} \varphi_{j}^{i}=0, \\
\varphi_{j}^{i} \xi^{j}=0, \quad \eta_{i} \xi^{i}=1,
\end{gathered}
$$

with respect to any local coordinate system $\left(U^{\prime}, x^{\prime i}\right)$ on $N$. A Riemannian metric $g$ on $N$ is associated, or compatible, to the almost contact structure $(\varphi, \xi, \eta)$ (and $(\varphi, \xi, \eta, g)$ is an almost contact metric structure on $N$ ) if

$$
g_{i j} \varphi_{k}^{i} \varphi_{\ell}^{j}=g_{k \ell}-\eta_{k} \eta_{\ell}, \quad g_{i j} \xi^{j}=\eta_{i} .
$$

Associated metrics always exist (cf. [13]). A contact metric structure is an almost contact metric structure $(\varphi, \xi, \eta, g)$ such that $\Omega=d \eta$, where $\Omega \in \Omega^{2}(N)$ is the 2-form given by $\Omega_{i j}=g_{i k} \varphi_{j}^{k}$.

Let $\phi: M \rightarrow N$ be a $C^{\infty}$ map from a strictly pseudoconvex CR manifold $M$ of CR dimension $n$ into a contact Riemannian manifold $(N, \varphi, \xi, \eta, g)$. Let $\theta$ be a contact form on $M$ such that the Levi form $G_{\theta}$ is positive definite. Let $H^{\prime}=\operatorname{Ker}(\eta)$ and let us consider the vector bundle valued form $(d \phi)_{H, H^{\prime}} \in \Gamma^{\infty}\left(H^{*} \otimes \phi^{-1} H^{\prime}\right)$ given by

$$
\left((d \phi)_{H, H^{\prime}}\right)_{x}=\Pi_{H^{\prime}, \phi(x)} \circ\left(d_{x} \phi\right): H_{x} \longrightarrow H_{\phi(x)}^{\prime}, \quad x \in M,
$$

where $\Pi_{H^{\prime}}: T(N) \rightarrow H^{\prime}$ is the natural projection associated to the decomposition $T(N)=H^{\prime} \oplus \mathbb{R} \xi$. Let $x \in M$ and let $\left\{X_{a}: 1 \leq a \leq 2 n\right\}$ be a local $G_{\theta}$-orthonormal frame of $H$ defined on an open neighborhood $U \subseteq M$ of $x \in U$. We set

$$
\begin{aligned}
& Q(\phi)_{x} \\
& =\left\|(d \phi)_{H, H^{\prime}}\right\|_{x}^{2} \\
& =\sum_{a=1}^{2 n} g_{\phi(x)}\left(\left((d \phi)_{H, H^{\prime}}\right)_{x} X_{a, x},\left((d \phi)_{H, H^{\prime}}\right)_{x} X_{a, x}\right) .
\end{aligned}
$$

Note that

$$
Q(\phi)=\operatorname{trace}_{G_{\theta}}\left\{\Pi_{H}\left(\phi^{*} g\right)\right\}-\left\|\Pi_{H} \phi^{*} \eta\right\|^{2} .
$$

Definition 1. Let $p \in(0,+\infty)$. A $C^{\infty}$ map $\phi: M \rightarrow N$ is said to be contact $p$-harmonic if $\phi$ is a critical point of the energy functional

$$
E_{\Omega, p}(\phi)=\int_{\Omega}\left\|(d \phi)_{H, H^{\prime}}\right\|^{p} \theta \wedge(d \theta)^{n}
$$

for any relatively compact domain $\Omega \subseteq M$. Contact 2harmonic maps are called contact harmonic maps.

Let $\nabla$ be the Tanaka-Webster connection of $(M, \theta)$ that is the unique linear connection on $M$ obeying to (i) $H$ is $\nabla$ parallel (i.e., $\nabla_{X} Y \in H$ for any $X \in \mathfrak{X}^{\infty}(M)$ and any $Y \in H$ ), (ii) $\nabla J=0$ and $\nabla g_{\theta}=0$, and (iii) the torsion tensor field $T_{\nabla}$ of $\nabla$ is pure (i.e., $T_{\nabla}(Z, W)=0, T_{\nabla}(Z, \bar{W})=2 i G_{\theta}(Z, \bar{W}) T$ for any $Z, W \in T_{1,0}(M)$ and $\tau \circ J+J \circ \tau=0$, where $\tau(X)=T_{\nabla}(T, X)$ for any $X \in \mathfrak{X}^{\infty}(M)$ (cf. Theorem 1.3 and Definition 1.25 in [9, pages 25-26]). The vector valued 1 -form $\tau$ is the pseudohermitian torsion of $\nabla$. Let $\nabla^{\prime}$ be the generalized Tanaka-Webster connection of $(N, \eta, g)$ given locally by

$$
\Gamma_{j k}^{\prime i}=\Gamma_{j k}^{i}+\eta_{j} \varphi_{k}^{i}-\eta_{k} \nabla_{j} \xi^{i}+\xi^{i} \nabla_{j} \eta_{k}
$$

(cf., e.g., [16]), where $\Gamma_{j k}^{i}$ are the Christoffel symbols of $g_{i j}$. Covariant derivatives are meant with respect to the LeviCivita connection of $(M, g)$. For each $X \in \mathfrak{X}^{\infty}(M)$ we consider $\phi_{*} X \in \Gamma^{\infty}\left(\phi^{-1} T N\right)$ given by

$$
\left(\phi_{*} X\right)(x)=\left(d_{x} \phi\right) X_{x} \in T_{\phi(x)}(N)=\left(\phi^{-1} T N\right)_{x}, \quad x \in M .
$$


Let $\nabla^{\phi}=\phi^{-1} \nabla^{\prime}$ be the connection induced by $\nabla^{\prime}$ in the pullback bundle $\phi^{-1} T N \rightarrow M$. We set

$$
\beta_{\phi}(X, Y)=\nabla_{X}^{\phi} \phi_{*} Y-\phi_{*} \nabla_{X} Y, \quad X, Y \in \mathfrak{X}^{\infty}(M) .
$$

Let $x \in M$ and let $\left\{X_{a}: 1 \leq a \leq 2 n\right\}$ be a local $G_{\theta^{-}}$ orthonormal frame of $H$ defined on an open neighborhood $U$ of $x$. We define a $C^{\infty}$ section $\Gamma(\phi)$ in $\phi^{-1} T N \rightarrow M$ by setting

$$
\Gamma(\phi)_{x}=\operatorname{trace}_{G_{\theta}}\left\{\Pi_{H} \beta_{\phi}\right\}_{x}=\sum_{a=1}^{2 n} \beta_{\phi}\left(X_{a}, X_{a}\right)_{x}
$$

where $\Pi_{H} \beta_{\phi}$ denotes the restriction of $\beta_{\phi}$ to $H \otimes H$. By a result in [10] the Euler-Lagrange equations associated to the variational principle $\delta E_{\Omega, p}(\phi)=0$ are

$$
\begin{gathered}
Q(\phi)^{-(p-2) / 2}\left[\left(\varphi^{2}\right)_{j}^{i} \circ \phi\right] \operatorname{div}\left(Q(\phi)^{(p-2) / 2} \nabla^{H} \phi^{j}\right) \\
=\operatorname{trace}_{G_{\theta}}\left\{\Pi_{H} \phi^{*}\left(\eta \otimes \tau_{N}\right)\right\} \\
-\sum_{a=1}^{2 n}\left[\left(\varphi^{2}\right)_{j}^{i} \circ \phi\right]\left(\Gamma_{k \ell}^{j} \circ \phi\right) X_{a}\left(\phi^{k}\right) X_{a}\left(\phi^{\ell}\right), \\
\operatorname{trace}_{G_{\theta}}\left\{\Pi_{H} \phi^{*} A_{N}\right\}=0,
\end{gathered}
$$

here $\varphi^{2}=-I+\eta \otimes \xi$ (cf., e.g., [13]). Also $\tau_{N}$ is the pseudohermitian torsion of $(N, \varphi, \xi, \eta, g)$; that is, $\tau_{N}(X)=$ $T_{\nabla^{\prime}}(\xi, X)$, and $A_{N}(X, Y)=g\left(\tau_{N} X, Y\right)$ for any $X, Y \in \mathfrak{X}^{\infty}(N)$. $\Gamma_{j k}^{\prime i}$ are again the local coefficients of $\nabla^{\prime}$ with respect to $\left(U^{\prime}, x^{\prime i}\right)$. In particular if $g$ is a Sasakian metric, then $\phi: M \rightarrow$ $N$ is contact $p$-harmonic if and only if

$$
\begin{aligned}
& {\left[\left(\varphi^{2}\right)_{j}^{i} \circ \phi\right]} \\
& \times\left\{\operatorname{div}\left(Q(\phi)^{(p-2) / 2} \nabla^{H} \phi^{j}\right)\right. \\
& \left.+Q(\phi)^{(p-2) / 2} \sum_{a=1}^{2 n}\left(\Gamma_{k \ell}^{\prime j} \circ \phi\right) X_{a}\left(\phi^{k}\right) X_{a}\left(\phi^{\ell}\right)\right\}=0, \\
& 1 \leq i \leq 2 m-1 .
\end{aligned}
$$

\section{Weak Contact Harmonic Maps}

Sections 3 and 4 are devoted to the study of local properties of weak critical points of the functional (15). A study of the regularity of weak solutions to subelliptic systems (such as (53)) was started by Wang [8], and Capogna and Garofalo [22], though only for maps from Carnot groups, (cf. also Zhou [23]).

Let $M$ be a strictly pseudoconvex CR manifold and $\theta$ a contact form on $M$. Let $\left\{X_{a}: 1 \leq a \leq 2 n\right\}$ be a local $G_{\theta^{-}}$ orthonormal frame of $H$ defined on the open set $U \subseteq M$ and $X_{a}^{*}$ the formal adjoint of $X_{a}$; that is,

$$
X_{a}^{*} u=-X_{a} u-f_{a} u, \quad u \in C_{0}^{1}(U),
$$

where $f_{a}=\partial b_{a}^{A} / \partial x^{A}+b_{a}^{B} \Gamma_{A B}^{A}$ and $X_{a}=b_{a}^{A} \partial / \partial x^{A}$. Also $\Gamma_{A B}^{C}$ are the local coefficients of the Tanaka-Webster connection of $(M, \theta)$ with respect to the local coordinate system $\left(U, x^{A}\right)$ on $M$. Clearly $\left(X_{a}^{*} u, v\right)=\left(u, X_{a} v\right)$ for any $u \in C_{0}^{1}(U)$, where $(u, v)=\int_{U} u \bar{v} d v$.

Proposition 2. Let $\phi: M \rightarrow N$ be a smooth map and $g$ a Sasakian metric on $N$. Then $\phi$ is contact p-harmonic if and only if

$$
\begin{aligned}
& {\left[\left(\varphi^{2}\right)_{j}^{i} \circ \phi\right]} \\
& \times \sum_{a=1}^{2 n}\left\{-X_{a}^{*}\left(Q(\phi)^{(p-2) / 2} X_{a} \phi^{j}\right)\right. \\
& \left.\quad+Q(\phi)^{(p-2) / 2}\left(\Gamma_{k \ell}^{\prime j} \circ \phi\right) X_{a}\left(\phi^{k}\right) X_{a}\left(\phi^{\ell}\right)\right\}=0
\end{aligned}
$$

for any local orthonormal frame $\left\{X_{a}: 1 \leq a \leq 2 n\right\}$ of $H$.

Proof. Let us note that $\operatorname{div}\left(X_{a}\right)=\operatorname{trace}\left\{\partial_{A} \mapsto \nabla_{\partial_{A}} X_{a}\right\}=f_{a}$, where $\partial_{A}=\partial / \partial x^{A}$. Thus (by (22))

$$
\operatorname{div}\left(Q(\phi)^{(p-2) / 2} \nabla^{H} \phi^{i}\right)=-\sum_{a=1}^{2 n} X_{a}^{*}\left(Q(\phi)^{(p-2) / 2} X_{a} \phi^{i}\right)
$$

on $U$. Then (23) follows from (21).

Example 3 (contact $p$-harmonic maps into the Heisenberg group). Let $N=\mathbb{U}_{m-1}, m \geq 2$, be the Heisenberg group (cf., e.g., [9, pages 11-14]). Let $\left(x^{\alpha}, y^{\alpha}, t\right)$ be the Cartesian coordinates on $\mathbb{R}^{2 m-1}$ and let

$$
\begin{array}{r}
X_{\alpha}=\frac{\partial}{\partial x^{\alpha}}+2 y^{\alpha} \frac{\partial}{\partial t}, \quad Y_{\alpha}=\frac{\partial}{\partial y^{\alpha}}-2 x^{\alpha} \frac{\partial}{\partial t}, \\
1 \leq \alpha \leq m-1 .
\end{array}
$$

Let $\varphi$ be the $(1,1)$-tensor field on $\mathbb{H}_{m-1}$ determined by

$$
\varphi\left(X_{\alpha}\right)=Y_{\alpha}, \quad \varphi\left(Y_{\alpha}\right)=-X_{\alpha}, \quad \varphi(\xi)=0,
$$

where $\xi=-\partial / \partial t$. Next the differential 1-form $\eta \in \Omega^{1}\left(\mathbb{H}_{m-1}\right)$ given by

$$
\eta=2 \sum_{\alpha=1}^{2 m-2}\left(y^{\alpha} d x^{\alpha}-x^{\alpha} d y^{\alpha}\right)-d t
$$

is a contact form on $\mathbb{U}_{m-1}$; that is, $\eta \wedge(d \eta)^{m-1}$ is a volume form. Let $H=\operatorname{Ker}(\eta)$. Finally we shall need the Riemannian metric $g$ on $\mathbb{U}_{m-1}$ given by $g=-d \eta(\cdot, \varphi \cdot)$ on $H \otimes H, g(\cdot, \xi)=$ 0 on $H$, and $g(\xi, \xi)=1$. Then $g$ is a Sasakian metric on $\mathbb{U}_{m-1}$ (and actually $\left(\mathbb{U}_{m-1}, g\right)$ is a Sasakian space form of $\varphi$-sectional -3 ; cf., e.g., [13]). A calculation shows that

$$
\varphi^{2}:\left(\begin{array}{ccc}
-\delta_{\beta}^{\alpha} & 0 & 0 \\
0 & -\delta_{\beta}^{\alpha} & 0 \\
-2 y_{\beta} & 2 x_{\beta} & 0
\end{array}\right),
$$


where $x_{\alpha}=x^{\alpha}$ and $y_{\alpha}=y^{\alpha}$. Let $T_{\alpha}=X_{\alpha}-i Y_{\alpha}$ and let $T_{1,0}\left(\mathbb{V}_{m-1}\right)_{x}$ be the span of $\left\{T_{\alpha}(x): 1 \leq \alpha \leq m-1\right\}$ over $\mathbb{C}$. Then $T_{1,0}\left(\mathbb{H}_{m-1}\right)$ is a strictly pseudoconvex CR structure on $\mathbb{H}_{m-1}$ and $\theta=-\eta$ is a contact form such that the Levi form $G_{\theta}$ is positive definite. Let $\nabla^{\prime}$ be the Tanaka-Webster connection of $\left(\mathbb{H}_{m-1}, \theta\right)$. A calculation shows that

$$
\begin{gathered}
\nabla_{\partial_{\alpha}}^{\prime} \partial_{\beta}=0, \quad \nabla_{\partial_{\alpha}}^{\prime} \partial_{\beta+m-1}=-2 \delta_{\alpha \beta} \xi, \\
\nabla_{\partial_{\alpha+m-1}}^{\prime} \partial_{\beta}=2 \delta_{\alpha \beta} \xi, \quad \nabla_{\partial_{\alpha+m-1}} \partial_{\beta+m-1}=0,
\end{gathered}
$$

where $\partial_{\alpha}=\partial / \partial x^{\alpha}$ and $\partial_{\alpha+m-1}=\partial / \partial y^{\alpha}$ for simplicity. Hence

$$
\Gamma_{\alpha, \beta+m-1}^{\prime 2 m-1}=-\Gamma_{\alpha+m-1, \beta}^{2 m-1}=2 \delta_{\alpha \beta}
$$

and the remaining connection coefficients vanish. The Webster metric $g$ of $\left(\mathbb{U}_{m-1}, \theta\right)$ is given by

$$
g:\left(\begin{array}{ccc}
2 \delta_{\alpha \beta}+4 y_{\alpha} y_{\beta} & -4 y_{\alpha} x_{\beta} & -2 y_{\alpha} \\
-4 x_{\alpha} y_{\beta} & 2 \delta_{\alpha \beta}+4 x_{\alpha} x_{\beta} & 2 x_{\alpha} \\
-2 y_{\beta} & 2 x_{\beta} & 1
\end{array}\right),
$$

hence (by a straightforward calculation)

$$
Q(\phi)=2 \sum_{a=1}^{2 n} \sum_{i=1}^{2 m-2}\left|X_{a}\left(\phi^{i}\right)\right|^{2}=2\left|X \phi^{\prime}\right|^{2}
$$

where $\phi=\left(\phi^{\prime}, \phi^{2 m-1}\right): M \rightarrow \mathbb{H}_{m-1}$ and $\phi^{\prime}=\left(\phi^{1}, \ldots\right.$, $\left.\phi^{2 m-2}\right)$. Let us substitute (28)-(32) into (23) so that to obtain

$$
\sum_{a=1}^{2 n} X_{a}^{*}\left(\left|X \phi^{\prime}\right|^{p-2} X_{a}\left(\phi^{i}\right)\right)=0, \quad 1 \leq i \leq 2 m-2 .
$$

Hence if $\phi: M \rightarrow \mathbb{M}_{2 m-1}$ is a contact $p$-harmonic map, then $\phi^{\prime}$ is subject to (33) while $\phi^{2 m-1}$ is an arbitrary function. Therefore, in general one may not expect regularity for a given (weak) contact $p$-harmonic map.

The identity (23) in Proposition 2 leads naturally to the notion of a weak solution to the contact $p$-harmonic map system. Indeed we may establish the following.

Lemma 4. A smooth map $\phi: M \rightarrow N$ of a strictly pseudoconvex CR manifold $M$ into a Sasakian manifold $N$ is contact p-harmonic if and only if

$$
\begin{aligned}
& \sum_{a=1}^{2 n}\left\{X_{a}^{*}\left(Q(\phi)^{(p-2) / 2}\left[\left(\varphi^{2}\right)_{j}^{i} \circ \phi\right] X_{a}\left(\phi^{j}\right)\right)\right. \\
& \left.\quad-Q(\phi)^{(p-2) / 2}\left[\left(\varphi^{2}\right)_{k}^{j} \circ \phi\right]\left(\Gamma_{j \ell}^{\prime i} \circ \phi\right) X_{a}\left(\phi^{k}\right) X_{a}\left(\phi^{\ell}\right)\right\} \\
& =0
\end{aligned}
$$

for any local orthonormal frame $\left\{X_{a}: 1 \leq a \leq 2 n\right\}$ of $H$ on $U$ and any local coordinate system $\left(U^{\prime}, x^{\prime i}\right)$ on $N$ such that $\phi^{-1}\left(U^{\prime}\right) \supseteq U$.
Proof. Let us multiply (23) by a test function $\psi \in C_{0}^{\infty}(U)$ and integrate by parts

$$
\begin{aligned}
& \int Q(\phi)^{(p-2) / 2} \sum_{a} X_{a}\left(\phi^{j}\right) X_{a}\left(\left(\varphi^{2}\right)_{j}^{i} \psi\right) d v \\
& =\int Q(\phi)^{(p-2) / 2} \sum_{a}\left(\varphi^{2}\right)_{j}^{i} \Gamma_{k \ell}^{\prime j} X_{a}\left(\phi^{k}\right) X_{a}\left(\phi^{\ell}\right) \psi d v .
\end{aligned}
$$

On the other hand (as both $\xi$ and $\eta$ are parallel with respect to $\nabla^{\prime}$ )

$$
\begin{gathered}
\frac{\partial \xi^{i}}{\partial x^{\prime k}}=-\Gamma_{k \ell}^{\prime i} \xi^{\ell}, \quad \frac{\partial \eta_{j}}{\partial x^{\prime k}}=\Gamma_{k j}^{\prime \ell} \eta_{\ell}, \\
\frac{\partial\left(\varphi^{2}\right)_{j}^{i}}{\partial x^{\prime k}}=\xi^{i} \eta_{\ell} \Gamma_{k j}^{\ell \ell}-\eta_{j} \xi^{\ell} \Gamma_{k \ell}^{\prime i}, \\
\left(\varphi^{2}\right)_{j}^{i} \Gamma_{k \ell}^{\prime i}+\eta_{\ell} \xi^{j} \Gamma_{j k}^{\prime i}-\xi^{i} \eta_{j} \Gamma_{k \ell}^{\prime j}=\left(\varphi^{2}\right)_{\ell}^{j} \Gamma_{j k}^{\prime i}-T_{k \ell}^{i},
\end{gathered}
$$

where $T_{k \ell}^{i}$ are the coefficients of $T_{\nabla^{\prime}}$ with respect to $\left(U^{\prime}, x^{\prime i}\right)$. Therefore (35) may be written as

$$
\begin{aligned}
& \int Q(\phi)^{(p-2) / 2} \\
& \times \sum_{a}\left\{\left(\varphi^{2}\right)_{j}^{i} X_{a}\left(\phi^{j}\right) X_{a}(\psi)\right. \\
&\left.\quad-\left(\varphi^{2}\right)_{k}^{j} \Gamma_{j \ell}^{i} X_{a}\left(\phi^{k}\right) X_{a}\left(\phi^{\ell}\right) \psi\right\} d v=0
\end{aligned}
$$

and Lemma 4 is proved.

Let us consider the function spaces

$$
W_{X}^{1, p}(U)=\left\{u \in L^{p}(U): X_{a} u \in L^{p}(U), 1 \leq a \leq 2 n\right\},
$$

where $X_{a} u$ are understood as weak derivatives. If $1 \leq p<\infty$, then $W_{X}^{1, p}(U)$ are separable Banach spaces with the norms

$$
\|u\|_{W_{X}^{1, p}(U)}=\left(\|u\|_{L^{p}(U)}^{p}+\sum_{a=1}^{2 n}\left\|X_{a} u\right\|_{L^{p}(U)}^{p}\right)^{1 / p} .
$$

Also $W_{X}^{1, p}(U)$ is reflexive provided that $1<p<\infty$. The central concept of this section may be introduced as follows. Let $\left\{X_{a}: 1 \leq a \leq 2 n\right\}$ be a $G_{\theta}$-orthonormal frame of $H$ defined on the open set $U \subseteq M$. Let $U^{\prime} \subseteq N$ be an open set which is relatively compact in a larger coordinate neighborhood in $N$.

Definition 5. A map $\phi: U \rightarrow U^{\prime}$ is said to be weak contact $p$ harmonic if it is a weak solution to (34); that is, $\phi^{j} \in W_{X}^{1, p}(U)$ for any $1 \leq j \leq 2 m-1$ and the identities (39) are satisfied for any test function $\psi \in C_{0}^{\infty}(U)$. (14)

Let $\phi: U \rightarrow U^{\prime}$ be a weak contact $p$-harmonic map. By

$$
Q(\phi)=\sum_{a}\left\{X_{a}\left(\phi^{i}\right) X_{a}\left(\phi^{j}\right)\left(g_{i j} \circ \phi\right)-\left[X_{a}\left(\phi^{i}\right)\left(\eta_{i} \circ \phi\right)\right]^{2}\right\}
$$


on $U$, hence

$$
\begin{gathered}
|Q(\phi)| \leq C|X \phi|^{2} \quad \text { a.e. in } U \\
|X \phi|^{2}=\sum_{a=1}^{2 n} \sum_{i=1}^{2 m-1}\left|X_{a}\left(\phi^{i}\right)\right|^{2}
\end{gathered}
$$

where $C=\max \left\{\sup _{\overline{U^{\prime}}}\left|g_{i j}\right|, \sup _{\overline{U^{\prime}}}\left|\eta_{i}\right|: 1 \leq i, j \leq 2 m-1\right\}$. Then both integrals in (39) are convergent and the adopted definition is legitimate.

Example 6 (Example 3 continued). A weak solution to (33) is a map $\phi=\left(\phi^{\prime}, \phi^{2 m-1}\right): U \rightarrow U^{\prime} \subset \subset \mathbb{M}_{m-1}$ such that $\phi^{\prime} \in W_{X}^{1, p}\left(U, \mathbb{R}^{2 m-2}\right)$ and

$$
\sum_{a=1}^{2 n} \int_{U}\left|X \phi^{\prime}\right|^{p-2} X_{a}\left(\phi^{i}\right) X_{a}(\psi) d v=0, \quad 1 \leq i \leq 2 m-2
$$

for any $\psi \in C_{0}^{\infty}(U)$. We need to recall the following general result, due to $\mathrm{Xu}$ and Zuily [24]. Let $X=\left\{X_{1}, \ldots, X_{m}\right\}$ be a Hörmander system on an open set $U \subseteq \mathbb{R}^{N}, N \geq 2$, and $\Omega \subset \mathbb{R}^{N}$ a domain such that $U \supset \bar{\Omega}$. Let $a^{i j}(x, y)$ be a symmetric and positive definite matrix defined in $\Omega \times \mathbb{R}^{v}$. If $|f(x, y, p)| \leq a|p|^{2}+b$ for any $(x, y, p) \in \Omega \times \mathbb{R}^{\nu} \times \mathbb{R}^{m \nu}$, then any continuous solution $\phi=\left(\phi^{1}, \ldots, \phi^{\nu}\right)$ to

$$
\begin{array}{r}
\sum_{i, j=1}^{m} X_{j}^{*}\left(a^{i j}(x, \phi(x)) X_{i} \phi^{\alpha}(x)\right)=f^{\alpha}(x, \phi(x), X \phi(x)), \\
1 \leq \alpha \leq \nu
\end{array}
$$

in $\Omega$ is actually smooth. Let us assume that $U$ is a domain such that $\bar{U}$ is contained in a coordinate neighborhood in $M$. By the result in [24] quoted above.

Proposition 7. For any weak solution $\phi=\left(\phi^{\prime}, \phi^{2 m}\right): U \rightarrow$ $U^{\prime} \subset \mathbb{H}_{m-1}$ to the contact $p$-harmonic map system (33) if $\phi^{\prime} \in$ $C^{0}\left(U, \mathbb{R}^{2 m-2}\right)$, then $\phi^{\prime} \in C^{\infty}\left(U, \mathbb{R}^{2 m-2}\right)$.

Of course in the particular case $p=2$ any distribution solution $\phi^{\prime}$ is $C^{\infty}$ (as the operator $\sum_{a=1}^{2 n} X_{a}^{*} X_{a}$ is hypoelliptic).

Example 8 (contact $p$-harmonic maps into the sphere). Let $N=S^{2 m-1} \subset \mathbb{R}^{2 m}$ and let $g$ be the canonical Sasakian metric on $S^{2 m-1}$. Then a $C^{\infty}$ contact $p$-harmonic map $\phi=$ $\left(\phi^{1}, \ldots, \phi^{2 m}\right): M \rightarrow S^{2 m-1}$ is a solution to

$$
\begin{aligned}
& {\left[\left(\varphi^{2}\right)_{j}^{i} \circ \phi\right] \sum_{a=1}^{2 n} X_{a}^{*}\left(Q(\phi)^{(p-2) / 2} X_{a} \phi^{j}\right)} \\
& =Q(\phi)^{(p-2) / 2} \\
& \quad \times\left\{\left[\left(\varphi^{2}\right)_{j}^{i} \circ \phi\right]|X \phi|^{2} \phi^{j}\right. \\
& \left.\quad+2 \sum_{a=1}^{2 n}\left(\phi^{*} \eta\right)\left(X_{a}\right)\left(\varphi_{j}^{i} \circ \phi\right) X_{a}\left(\phi^{j}\right)\right\},
\end{aligned}
$$

for any $1 \leq i \leq 2 m-1$. Here $|X \phi|^{2}=\sum_{\beta=1}^{2 m} \sum_{a=1}^{2 n}\left|X_{a} \phi^{\beta}\right|^{2}$ and $\sum_{\beta=1}^{2 m} \phi_{\beta}^{2}=1$ with $\phi_{\beta}=\phi^{\beta}, 1 \leq \beta \leq 2 m$. Equation (46) follows from (23) by computing the Christoffel symbols of $S^{2 m-1}$ with respect to the local coordinate system

$$
\begin{gathered}
\chi^{\prime}: U^{\prime} \rightarrow \mathbb{R}^{2 m-1}, \quad \chi^{\prime}(x)=x^{\prime}, \quad x=\left(x^{\prime}, x_{2 m}\right) \in U^{\prime}, \\
U^{\prime}=S^{2 m-1} \cap\left\{x_{2 m}>0\right\}, \quad x^{\prime}=\left(x_{1}, \ldots, x_{2 m-1}\right),
\end{gathered}
$$

that is

$$
\left|\begin{array}{c}
i \\
j k
\end{array}\right|=x^{i} g_{j k}, \quad g_{j k}=\delta_{j k}+\frac{x_{j} x_{k}}{1-\left|x^{\prime}\right|^{2}}
$$

so that

$$
\begin{aligned}
\sum_{a=1}^{2 n}\left(\left|\begin{array}{c}
i \\
j k
\end{array}\right| \circ \phi\right) X_{a}\left(\phi^{j}\right) X_{a}\left(\phi^{k}\right) & =|X \phi|^{2} \phi^{i} \\
1 & \leq i \leq 2 m-1 .
\end{aligned}
$$

On the other hand (cf. [9])

$$
\left|\begin{array}{c}
i \\
j k
\end{array}\right|=\Gamma_{j k}^{\prime i}+\omega_{j k} \xi^{i}+\eta_{j} \varphi_{k}^{i}+\eta_{k} \varphi_{j}^{i}
$$

so that

$$
\begin{aligned}
\left(\left|\begin{array}{c}
i \\
j k
\end{array}\right| \circ \phi\right) X_{a}\left(\phi^{j}\right) X_{a}\left(\phi^{k}\right) & \\
= & \left(\Gamma_{j k}^{i} \circ \phi\right) X_{a}\left(\phi^{j}\right) X_{a}\left(\phi^{k}\right) \\
& +2\left(\phi^{*} \eta\right)\left(X_{a}\right)\left(\varphi_{j}^{i} \circ \phi\right) X_{a}\left(\phi^{j}\right)
\end{aligned}
$$

for any Sasakian metric $g$. When $N=S^{2 m-1}$, the identities (49)-(51) lead to

$$
\begin{aligned}
& \sum_{a=1}^{2 n}\left(\Gamma_{j k}^{i} \circ \phi\right) X_{a}\left(\phi^{j}\right) X_{a}\left(\phi^{k}\right) \\
& \quad=|X \phi|^{2} \phi^{i}-\sum_{a=1}^{2 n} 2\left(\phi^{*} \eta\right)\left(X_{a}\right)\left(\varphi_{j}^{i} \circ \phi\right) X_{a}\left(\phi^{j}\right)
\end{aligned}
$$

and then to (46) by taking into account that $\varphi$ is an $f$ structure on $S^{2 m-1}$; that is, $\varphi^{3}+\varphi=0$. Our next purpose in this example is to prove the following result. 
Proposition 9. Let $\phi: \mathbb{U}_{n} \rightarrow S^{2 m-1}$ be a horizontal map. Then $\phi$ is contact $p$-harmonic if and only if $\phi$ is subelliptic $p$ harmonic with respect to the canonical Hörmander system $X=$ $\left\{X_{\gamma}, Y_{\gamma}: 1 \leq \gamma \leq n\right\}$ on $\mathbb{\boxplus}_{n}$.

According to [7] given a Hörmander system of vector fields $\left\{X_{a}\right\}$ defined on an open set $O \subseteq \mathbb{R}^{N}$, one may adopt the following.

Definition 10. A subelliptic $p$-harmonic map is a $C^{\infty}$ solution $\phi: O \rightarrow \mathbb{R}^{2 m}$ to the system (the formal adjoint of $X_{a}$ in [7] is $-X_{a}^{*}$ under the conventions adopted in the present paper)

$$
\sum_{a} X_{a}^{*}\left(|X \phi|^{p-2} X_{a} \phi^{\alpha}\right)=|X \phi|^{p} \phi^{\alpha}, \quad 1 \leq \alpha \leq 2 m,
$$

such that $\sum_{\alpha=1}^{2 m} \phi_{\alpha}^{2}=1$. that

A horizontal map is a smooth map $\phi: \mathbb{W}_{n} \rightarrow S^{2 m-1}$ such

$$
X_{a}\left(\phi^{i}\right)\left(\eta_{i} \circ \phi\right)=0, \quad 1 \leq a \leq 2 n .
$$

One may define weak solutions $\phi: \mathbb{H}_{n} \rightarrow U^{\prime}$ to (54) by requiring that $\phi^{i} \in W_{X}^{1, p}(U)$ for some $1 \leq p<\infty$ and that (54) holds a.e. in $U$. Then the statement in Proposition 9 holds for weak solutions of the relevant equations as well. In particular, by a result in [7], any weak horizontal contact $p$ harmonic map $\phi: \mathbb{H}_{n} \rightarrow U^{\prime}$ is locally Hölder continuous provided that $p \geq 2 n+2$.

The proof of Proposition 9 is to write (46) in the form (53). We need the following.

Lemma 11. Let $M$ be a strictly pseudoconvex CR manifold. A smooth map $\phi: M \rightarrow S^{2 m-1}$ is contact $p$-harmonic if and only if

$$
-\sum_{a=1}^{2 n} X_{a}^{*}\left(Q(\phi)^{(p-2) / 2}\left[\left(\varphi^{2}\right)_{j}^{i} \circ \phi\right] X_{a}\left(\phi^{j}\right)\right)=Q(\phi)^{p / 2} \phi^{i},
$$

for any $1 \leq i \leq 2 m-1$ and any local orthonormal frame $\left\{X_{a}\right.$ : $1 \leq a \leq 2 n\}$ of $H$.

By (14) if $\phi: M \rightarrow S^{2 m-1}$ is a horizontal map, then $Q(\phi)=|X \phi|^{2}$ and one may readily check that (55) is equivalent to (53) for any $1 \leq i \leq 2 m-1$. Of course the component $\phi_{2 m}$ will satisfy (53) as well (as a consequence of the constraint $\sum_{\alpha=1}^{2 n} \phi_{\alpha}^{2}=1$ ). To prove Lemma 11, let us multiply (46) by a test function $\psi \in C_{0}^{\infty}(U)$ and integrate over $U$. The left-hand side of the resulting equation is

$$
\begin{gathered}
\sum_{a} \int_{U}\left(\varphi^{2}\right)_{j}^{i} X_{a}^{*}\left(\rho X_{a}\left(\phi^{j}\right)\right) \psi d v \\
=\sum_{a} \int \rho X_{a}\left(\phi^{j}\right) X_{a}\left(\left(\varphi^{2}\right)_{j}^{i} \psi\right) d v \\
=\sum_{a} \int \rho\left\{X_{a}\left(\phi^{j}\right)\left(\varphi^{2}\right)_{j}^{i} X_{a}(\psi)\right. \\
\left.+\psi X_{a}\left(\phi^{j}\right) X_{a}\left(\left(\varphi^{2}\right)_{j}^{i} \circ \phi\right)\right\} d v \\
=\sum_{a} \int\left\{X_{a}^{*}\left(\rho\left[\left(\varphi^{2}\right)_{j}^{i} \circ \phi\right] X_{a}\left(\phi^{j}\right)\right) \psi\right. \\
\left.+\rho \psi X_{a}\left(\phi^{j}\right) X_{a}\left(\phi^{k}\right) \frac{\partial\left(\varphi^{2}\right)_{j}^{i}}{\partial x^{\prime k}}\right\} d v,
\end{gathered}
$$

where $\rho=Q(\phi)^{(p-2) / 2}$. Then (by (37))

$$
\begin{aligned}
\sum_{a} & X_{a}\left(\phi^{j}\right) X_{a}\left(\phi^{k}\right) \frac{\partial\left(\varphi^{2}\right)_{j}^{i}}{\partial x^{\prime k}} \\
= & \sum_{a} X_{a}\left(\phi^{j}\right) X_{a}\left(\phi^{k}\right)\left(\xi^{i} \eta_{\ell} \Gamma_{k j}^{\ell}-\eta_{j} \xi^{\ell} \Gamma_{k \ell}^{\prime i}\right) \\
= & (\text { by (52) and (50)) } \\
= & \xi^{i} \eta_{\ell}\left(|X \phi|^{2} \phi^{\ell}-2 \sum_{a} \eta_{j} X_{a}\left(\phi^{j}\right) \varphi_{k}^{\ell} X_{a}\left(\phi^{k}\right)\right) \\
& -\sum_{a} X_{a}\left(\phi^{j}\right) X_{a}\left(\phi^{k}\right) \eta_{j}\left(\left|\begin{array}{c}
i \\
k \ell
\end{array}\right| \xi^{\ell}-\varphi_{k}^{i}\right) \\
= & \xi^{i} \eta_{\ell} \phi^{\ell}|X \phi|^{2} \\
& +\sum_{a}\left\{\eta_{j} X_{a}\left(\phi^{j}\right) \varphi_{k}^{i} X_{a}\left(\phi^{k}\right)-X_{a}\left(\phi^{j}\right) X_{a}\left(\phi^{k}\right) \eta_{j} \eta_{k} \phi^{i}\right\},
\end{aligned}
$$

hence (46) implies

$$
\begin{aligned}
\sum_{a} X_{a}^{*}\left(Q(\phi)^{(p-2) / 2}\left[\left(\varphi^{2}\right)_{j}^{i} \circ \phi\right] X_{a}\left(\phi^{j}\right)\right) \\
=Q(\phi)^{(p-2) / 2}\left(-|X \phi|^{2}+\sum_{a}\left[X_{a}\left(\phi^{j}\right)\left(\eta_{j} \circ \phi\right)\right]^{2}\right) \phi^{i}
\end{aligned}
$$

which yields (55) because on the sphere

$$
Q(\phi)=|X \phi|^{2}-\left\|\Pi_{H} \phi^{*} \eta\right\|^{2} .
$$

Lemma 11 is proved.

The notion of a weak contact harmonic map as introduced above is confined to maps $\phi: M \rightarrow N$ such that the target contact Riemannian manifold $N$ is covered by a single coordinate neighborhood. Another natural approach (customary in 
the theory of harmonic maps among Riemannian manifolds, cf., e.g., [4, page 38]) is to use Nash's embedding theorem (cf. [25]) in order to embed isometrically the target manifold $N$ into some Euclidean space $\mathbb{R}^{K}$ and produce an alternative first variation formula (cf. Theorem 2.22 in [26, page 139]) depending however on the embedding $N \hookrightarrow \mathbb{R}^{K}$.

A generalization of Nash's embedding theorem to the context of contact Riemannian geometry has been obtained by D'Ambra [27]. Let $\mathbb{H}_{L} \approx \mathbb{C}^{L} \times \mathbb{R}$ be the Heisenberg group equipped with the standard Sasakian structure $\left(\varphi_{0}, \xi_{0}, \eta_{0}, g_{0}\right)$. Let $(N,(\varphi, \xi, \eta, g))$ be a contact Riemannian manifold. By a result in [27], if $N$ is compact and $L \geq \operatorname{dim}(N)+1$, there is a $C^{1}$-embedding $\iota: N \rightarrow \mathbb{H}_{L}$ which is both horizontal, that is, $\iota_{*} H^{\prime} \subset \iota^{-1} \operatorname{Ker}\left(\eta_{0}\right)$, and isometric in the sense that $\iota$ preserves the Levi forms

$$
g_{p}(v, w)=g_{0, \iota(p)}\left(\left(d_{p} \iota\right) v,\left(d_{p} \iota\right) w\right), \quad v, w \in H_{p}^{\prime}, \quad p \in N .
$$

Any contact Riemannian manifold $N$ is in particular a sub-Riemannian manifold (in the sense of [28]); hence $N$ carries the Carnot-Carathéodory metric $d_{N}: N \times N \rightarrow$ $[0,+\infty)$ associated to the sub-Riemannian structure $\left(H^{\prime}, g\right)$. In particular $\iota$ is an isometry among the metric spaces $\left(N, d_{N}\right)$ and $\left(\mathbb{H}_{L}, d_{X}\right)$ (cf. Section 7 for the definition of the distance function $\left.d_{X}: \mathbb{H}_{L} \times \mathbb{H}_{L} \times[0,+\infty)\right)$. As $\mathbb{H}_{L}$ also possesses a linear space structure, the methods in [29] (methods of direct infinitesimal geometry) become available on a contact Riemannian manifold (e.g., one may merely use the balls with respect to $d_{N}$ and the linear structure of the ambient space $\mathbb{H}_{L}$ to reformulate on $N$ Definition 2.1 in [29, page 280]) and we conjecture that the arguments in [29] may be recovered to study the equation $\Delta_{b} u=0$ on a strictly pseudoconvex CR manifold (the theory in [29] only deals with second order degenerate elliptic equations on domains in $\mathbb{R}^{n}$ ). Unfortunately the existence of $C^{1}$-embeddings of given contact structures is not sufficient for differential geometric purposes, as long as Gauss and Weingarten formulae (which require two derivatives of $\iota$ ) are involved. The problem of improving D'Ambra's proof (to get a horizontal embedding of class at least $C^{2}$ ) is open.

\section{Contact Harmonic Maps into Spheres}

Let $\Omega \subset \mathbb{R}^{N}$ be a bounded open set and $X=\left\{X_{1}, \ldots, X_{m}\right\}$ a Hörmander system of vector fields $X_{a}=b_{a}^{A}(x) \partial / \partial x^{A} \epsilon$ $\mathfrak{X}\left(\mathbb{R}^{N}\right)$ such that $b_{a}^{A} \in C^{\infty}\left(\mathbb{R}^{N}\right) \cap \operatorname{Lip}\left(\mathbb{R}^{N}\right)$. We recall (cf., e.g., $[9$, page 261$])$ the following.

Definition 12. A number $D$ is a homogeneous dimension relative to $\Omega$ with respect to $X$ if there is a constant $C>0$ such that

$$
\frac{\left|B_{X}(x, r)\right|}{\left|B_{X}\left(x_{0}, r_{0}\right)\right|} \geq C\left(\frac{r}{r_{0}}\right)^{D}
$$

for any Carnot-Carathéodory ball $B_{0}=B_{X}\left(x_{0}, r_{0}\right)$ of center $x_{0} \in \Omega$ and radius $0<r_{0} \leq \operatorname{diam}(\Omega)$ and any CarnotCarathéodory ball $B=B_{X}(x, r)$ of center $x \in B_{0}$ and radius $0<r \leq r_{0}$.
The diameter of $\Omega$ is meant with respect to the CarnotCarathéodory metric associated to $X$. Hajłasz and Strzelecki [7] studied local properties of weak solutions to the system (53). Their main finding is that every weak subelliptic $D$ harmonic map $\phi \in W_{X}^{1, D}\left(\Omega, S^{\nu}\right)$ (i.e., every weak solution to (53) with $p=D$ ) is locally Hölder continuous. Maps $\phi: \Omega \rightarrow$ $S^{\nu}$ with values in a unit sphere $S^{\nu} \subset \mathbb{R}^{\nu+1}$ have a special status due to the fact that the subelliptic harmonic map system (here (53)) may be written in a simple form using an approach commonly referred to as the Frédéric Hélein trick (cf. [7, page 353], see also Hélein [30]). The purpose of this section is to start a study of weak solutions to the system (55) following the ideas in [7] though confined to maps $\phi: \mathbb{H}_{n} \rightarrow S^{2 m-1}$ which are "close to horizontal" in a sense to be made precise in the sequel.

Let $\mathbb{U}_{n}$ be the Heisenberg group equipped with the standard contact form $\theta=d t+i \sum_{\gamma=1}^{n}\left(z^{\gamma} d \bar{z}_{\gamma}-\bar{z}_{\gamma} d z^{\gamma}\right)$. Let $U \subseteq \mathbb{M}_{n}$ be a bounded domain. Let $\left\{X_{a}: 1 \leq a \leq 2 n\right\}=$ $\left\{X_{\gamma}, Y_{\gamma}: 1 \leq \gamma \leq n\right\}$ be the $G_{\theta}$-orthonormal frame given by $X_{\gamma}=\partial / \partial x^{\gamma}+2 y^{\gamma} T$ and $Y_{\gamma}=\partial / \partial y^{\gamma}-2 x^{\gamma} T$, where $T=\partial / \partial t$ as in Example 3. Clearly the coefficients of the $X_{a}$ 's lie in $C^{\infty}\left(\mathbb{R}^{2 n+1}\right) \cap \operatorname{Lip}\left(\mathbb{R}^{2 n+1}\right)$. We recall that an absolutely continuous curve $\gamma:[0, \tau] \rightarrow \mathbb{U}_{n}$ is admissible if

$$
\frac{d \gamma}{d t}(t)=\sum_{a=1}^{2 n} u_{a}(t) X_{a}(\gamma(t))
$$

for some functions $u_{a}(t)$ such that $\sum_{a=1}^{2 n} u_{a}(t)^{2} \leq 1$.

Definition 13. The Carnot-Carathéodory distance $d_{X}(x, y)$ among two points $x, y \in \mathbb{H}_{n}$ is the infimum of all $\tau>0$ for which there exists an admissible curve $\gamma:[0, \tau] \rightarrow \mathbb{U}_{n}$ such that $\gamma(0)=x$ and $\gamma(\tau)=y$. Balls with respect to $d_{X}: \mathbb{H}_{n} \times \mathbb{H}_{n} \rightarrow[0,+\infty)$ are denoted by $B_{X}(x, r)=\{y \in$ $\left.\mathbb{H}_{n}: d_{X}(x, y)<r\right\}$ and referred to as Carnot-Carathéodory balls.

We shall characterize horizontal maps in terms of the first order differential operator

$$
L_{a} u=u^{m+\alpha} X_{a}\left(u_{\alpha}\right)-u^{\alpha} X_{a}\left(u_{m+\alpha}\right)
$$

defined for $u=\left(u_{1}, \ldots, u_{2 m}\right) \in W_{X}^{1, p}\left(U, \mathbb{R}^{2 m}\right)$.

Proposition 14. Let $\phi: U \rightarrow U^{\prime}=S^{2 m-1} \cap\left\{x_{2 m}>0\right\} \subset \mathbb{R}^{2 m}$ be a map such that $\phi_{A} \in W_{X}^{1, p}(U)$ for any $1 \leq A \leq 2 m$. Then $\phi: U \rightarrow U^{\prime}$ is a (weak) horizontal map if and only if $L_{a} \phi=0$ for any $1 \leq a \leq 2 n$.

Let $\left(z_{1}, \ldots, z_{m}\right)$ be the natural complex coordinates on $\mathbb{C}^{m}$ and set $z_{\alpha}=x_{\alpha}+i y_{\alpha}$ and $\left(x_{1}, \ldots, x_{2 m}\right)=$ $\left(x_{1}, \ldots, x_{m}, y_{1}, \ldots, y_{m}\right)$. The following conventions are adopted as to the range of indices:

$$
\begin{array}{cc}
1 \leq A, B, \cdots \leq 2 m, & 1 \leq i, j, \cdots \leq 2 m-1, \\
1 \leq \alpha, \beta, \cdots \leq m, & 1 \leq r, s, \cdots \leq m-1 .
\end{array}
$$

Let $v=x^{\alpha} \partial / \partial x^{\alpha}+y^{\alpha} \partial / \partial y^{\alpha} \in \mathfrak{X}^{\infty}\left(\mathbb{R}^{2 m}\right)$ so that the pointwise restriction of $\nu$ to $S^{2 m-1}$ is a unit normal field on $S^{2 m-1}$. Let $J_{0}$ 
be the complex structure on $\mathbb{C}^{m}$. Then $\xi \in \mathfrak{X}^{\infty}\left(S^{2 m-1}\right)$ given by $\left(d_{x} l\right) \xi_{x}=J_{0, x} v_{x}$ for any $x \in S^{2 m-1}$ is the Reeb vector field on $S^{2 m-1}$. Here $\iota: S^{2 m-1} \rightarrow \mathbb{R}^{2 m}$ is the inclusion. With respect to the local chart $\chi^{\prime}=\left(x_{1}, \ldots, x_{2 m-1}\right)$ in Example 8 the Reeb vector $\xi$ is given by

$$
\xi^{\alpha}=-y^{\alpha}, \quad \xi^{m+r}=x^{r} .
$$

Then $\eta_{i}=g_{i j} \xi^{j}$ together with (48) in Example 8 leads to

$$
\eta_{\alpha}=-y_{\alpha}-\frac{x_{m}}{y_{m}} x_{\alpha}, \quad \eta_{m+r}=x_{r}-\frac{x_{m}}{y_{m}} y_{r} .
$$

Finally (66) implies that $X_{a}\left(\phi^{i}\right)\left(\eta_{i} \circ \phi\right)=-L_{a} \phi$. Proposition 14 is proved. In particular $Q(\phi)$ may be written as

$$
Q(\phi)=|X \phi|^{2}-\sum_{a=1}^{2 n}\left|L_{a} \phi\right|^{2} .
$$

Our next task is to put (55) into a more tractable form.

Proposition 15. Let $\phi=\left(\phi_{1}, \ldots, \phi_{2 m}\right): U \rightarrow U^{\prime}$ such that $\phi_{A} \in W_{X}^{1, p}(U)$. Let us consider the functions

$$
\begin{gathered}
V_{\alpha, a}=Q(\phi)^{(p-2) / 2}\left\{X_{a}\left(\phi_{\alpha}\right)-\phi_{m+\alpha} L_{a} \phi\right\}, \\
V_{n+\alpha, a}=Q(\phi)^{(p-2) / 2}\left\{X_{a}\left(\phi_{m+\alpha}\right)+\phi_{\alpha} L_{a} \phi\right\},
\end{gathered}
$$

with $1 \leq \alpha \leq m$. Let $V_{A}=\left(V_{A, 1}, \ldots, V_{A, 2 n}\right)$ for any $1 \leq A \leq$ $2 m$. Then $\phi: U \rightarrow U^{\prime}$ is a contact p-harmonic map if and only if

$$
X^{*} \cdot V_{A}=Q(\phi)^{p / 2} \phi_{A}, \quad 1 \leq A \leq 2 m .
$$

Here the dot product means $X^{*} \cdot V_{A}=\sum_{a=1}^{2 n} X_{a}^{*}\left(V_{A, a}\right)$. Using $\varphi^{2}=-I+\eta \otimes \xi$ and (65) and (66), one obtains

$$
\begin{aligned}
& {\left[\left(\varphi^{2}\right)_{j}^{i}\right]_{1 \leq i, j \leq 2 m-1}} \\
& \quad=\left[\begin{array}{cc}
-\delta_{\beta}^{\alpha}+y^{\alpha}\left(y_{\beta}+\frac{x_{m}}{y_{m}} x_{\beta}\right) & -y^{\alpha}\left(x_{r}-\frac{x_{m}}{y_{m}} y_{r}\right) \\
-x^{s}\left(y_{\beta}+\frac{x_{m}}{y_{m}} x_{\beta}\right) & -\delta_{r}^{s}+x^{s}\left(x_{r}-\frac{x_{m}}{y_{m}} y_{r}\right)
\end{array}\right] .
\end{aligned}
$$

Then substitution into (55) leads to

$$
\begin{aligned}
& \sum_{a=1}^{2 n} X_{a}^{*}\left[Q(\phi)^{(p-2) / 2}\left(X_{a}\left(\phi^{\alpha}\right)-\phi^{m+\alpha} L_{a} \phi\right)\right]=Q(\phi)^{p / 2} \phi^{\alpha} \\
& \sum_{a=1}^{2 n} X_{a}^{*}\left[Q(\phi)^{(p-2) / 2}\left(X_{a}\left(\phi^{m+s}\right)+\phi^{s} L_{a} \phi\right)\right]=Q(\phi)^{p / 2} \phi^{m+s} .
\end{aligned}
$$

It remains to be shown that (71) and (72) imply

$$
\sum_{a=1}^{2 n} X_{a}^{*}\left[Q(\phi)^{(p-2) / 2}\left(X_{a}\left(\phi^{2 m}\right)+\phi^{m} L_{a} \phi\right)\right]=Q(\phi)^{p / 2} \phi^{2 m} .
$$

Let us multiply (71) by $\phi^{\beta} \psi$, where $\psi \in C_{0}^{\infty}(U)$ is an arbitrary test function, and integrate over $U$ so that to obtain (after integration by parts)

$$
\begin{gathered}
\sum_{a} X_{a}^{*}\left[Q(\phi)^{(p-2) / 2}\left(X_{a}\left(\phi^{\alpha}\right)-\phi^{m+\alpha} L_{a} \phi\right) \phi^{\beta}\right] \\
=Q(\phi)^{(p-2) / 2} \\
\times\left\{Q(\phi) \phi^{\alpha} \phi^{\beta}-\sum_{a} X_{a}\left(\phi^{\alpha}\right) X_{a}\left(\phi^{\beta}\right)\right. \\
\left.\quad+\sum_{a} \phi^{m+\alpha} X_{a}\left(\phi^{\beta}\right) L_{a} \phi\right\} .
\end{gathered}
$$

Similarly let us multiply (72) by $\phi^{m+r} \psi$ so that to obtain

$$
\begin{aligned}
\sum_{a} X_{a}^{*}\left[Q(\phi)^{(p-2) / 2}\left(X_{a} \phi^{m+s}+\phi^{s} L_{a} \phi\right) \phi^{m+r}\right] \\
=Q(\phi)^{(p-2) / 2} \\
\times\left\{Q(\phi) \phi^{m+s} \phi^{m+r}-\sum_{a} X_{a}\left(\phi^{m+s}\right) X_{a}\left(\phi^{m+r}\right)\right. \\
\left.\quad-\sum_{a} \phi^{s} X_{a}\left(\phi^{m+r}\right) L_{a} \phi\right\} .
\end{aligned}
$$

Let us contract the indices $\alpha$ and $\beta$ in (74) (resp., $r$ and $s$ in (75)), add the resulting equations, and use the identities

$$
\begin{gathered}
X_{a}\left(\phi^{\alpha}\right) \phi_{\alpha}+X_{a}\left(\phi^{m+r}\right) \phi_{m+r}=-X_{a}\left(\phi^{2 m}\right) \phi_{2 m}, \\
-\phi^{m+\alpha} \phi_{\alpha}+\phi^{r} \phi_{m+r}=-\phi_{m} \phi_{2 m}, \\
\phi^{\alpha} \phi_{\alpha}+\phi^{m+r} \phi_{m+r}=1-\phi_{2 m}^{2}, \\
\phi^{m+\alpha} X_{a}\left(\phi_{\alpha}\right)-\phi^{r} X_{a}\left(\phi_{m+r}\right)=L_{a} \phi+\phi^{m} X_{a}\left(\phi_{2 m}\right) .
\end{gathered}
$$

We get

$$
\begin{aligned}
-\sum_{a} X_{a}^{*}\left[Q(\phi)^{(p-2) / 2}\left(X_{a}\left(\phi^{2 m}\right)+\phi^{m} L_{a} \phi\right) \phi_{2 m}\right] \\
=Q(\phi)^{(p-2) / 2}\left\{Q(\phi)\left(1-\phi_{2 m}^{2}\right)-\sum_{a} X_{a}\left(\phi^{i}\right) X_{a}\left(\phi_{i}\right)\right. \\
\left.+\sum_{a}\left[L_{a} \phi+\phi^{m} X_{a}\left(\phi_{2 m}\right)\right] L_{a} \phi\right\} .
\end{aligned}
$$

Let us use $Q(\phi)-\sum_{a} X_{a}\left(\phi^{i}\right) X_{a}\left(\phi_{i}\right)+\sum_{a}\left(L_{a} \phi\right)^{2}=X_{a}\left(\phi_{2 m}\right)^{2}$ (a consequence of (67)). Finally

$$
\begin{aligned}
\sum_{a} X_{a}^{*} & {\left[Q(\phi)^{(p-2) / 2}\left(X_{a}\left(\phi^{2 m}\right)+\phi^{m} L_{a} \phi\right) \phi_{2 m}\right] } \\
= & Q(\phi)^{(p-2) / 2} \\
& \times\left\{Q(\phi) \phi_{2 m}^{2}-\sum_{a} X_{a}\left(\phi_{2 m}\right)^{2}-\sum_{a} \phi^{m} X_{a}\left(\phi_{2 m}\right) L_{a} \phi\right\} .
\end{aligned}
$$


Now the identity (73) follows from (78) and $X_{a}^{*}=-X_{a}-f_{a}$. Proposition 15 is proved.

The crucial manner of exploiting the constraint $\sum_{A=1}^{2 m} \phi_{A}^{2}=1$ is contained in the following.

Proposition 16. Let $U \subset \mathbb{H}_{n}$ be a bounded domain and $\phi$ : $U \rightarrow S^{2 m-1} \subset \mathbb{R}^{2 m}, \phi=\left(\phi_{1}, \ldots, \phi_{2 m}\right)$, a map such that $\phi_{A} \in$ $W_{X}^{1, p}(U)$. Then

$$
V_{A}=\sum_{B=1}^{2 m} \phi_{B} E_{A, B}
$$

where one has set $E_{A, B}=\phi_{B} V_{A}-\phi_{A} V_{B}$. Moreover if $\phi$ is a contact p-harmonic map, then

$$
\begin{aligned}
& X^{*} \cdot E_{A, B} \\
& \quad=Q(\phi)^{(p-2) / 2}\left\{\sigma_{B} \phi_{B+m} X\left(\phi_{A}\right)-\sigma_{A} \phi_{A+m} X\left(\phi_{B}\right)\right\} L \phi,
\end{aligned}
$$

where $\sigma_{A}=1$ if $1 \leq A \leq m, \sigma_{A}=-1$ if $m+1 \leq A \leq 2 m$, and the range of the indices in (80) is meant $\bmod m$.

The identity (79) is a consequence of the constraint alone. The identity (80) for $A=\alpha$ and $B=\beta$ follows from (74) (interchange $\alpha$ and $\beta$ in (74) and subtract the resulting identity from (74)). In general, for any $\psi \in C_{0}^{\infty}(U)$

$$
\begin{aligned}
\int_{U} X^{*} & \cdot\left(\phi_{A} V_{B}\right) \psi d v \\
& =\int_{U} V_{B} \cdot\left[X\left(\psi \phi_{A}\right)-\psi X\left(\phi_{A}\right)\right] d v \\
& =\int_{U}\left(X^{*} \cdot V_{B}\right) \phi_{A} \psi d v-\int_{U}\left[V_{B} \cdot X\left(\phi_{A}\right)\right] \psi d v,
\end{aligned}
$$

hence (by (69))

$$
\begin{aligned}
\int_{U} X^{*} & \cdot\left(\phi_{A} V_{B}\right) \psi d v \\
= & \int_{U}\left\{Q(\phi)^{p / 2} \phi_{B} \phi_{A}-Q(\phi)^{(p-2) / 2} X\left(\phi_{B}\right) \cdot X\left(\phi_{A}\right)\right\} \psi \\
& -\int_{U} Q(\phi)^{(p-2) / 2} \sigma_{B} \phi_{B+m}\left[(L \phi) \cdot X\left(\phi_{A}\right)\right] \psi d v
\end{aligned}
$$

Now let us interchange $A$ and $B$ in (82) to produce another identity of the sort and subtract it from (82). This yields (80). Proposition 16 is proved.

Although regularity of contact $p$-harmonic maps cannot be expected in general (cf. Example 3), a few fundamental questions may be asked. For instance, what is the the outcome of the ordinary hole filling argument (cf., e.g., [31, pages 3840]) and of Moser's iteration technique in regularity theory? our finding in this direction is Theorem 20. We shall need the following.

Lemma 17. Let $U \subset \mathbb{H}_{n}$ be a bounded domain. Let $R_{0}>0$ and $U_{1} \subset \subset U$ such that $B_{X}\left(x, 400 R_{0}\right) \subset U$ for any $x \in U_{1}$. Let
$\mathbb{B}=B_{X}\left(x_{0}, r\right)$ with $x_{0} \in U_{1}$ be a Carnot-Carathéodory ball such that $0<r \leq R_{0}$ and let $\psi \in W_{X}^{1,2 n+2}(\mathbb{B})$ be a function of compact support. Then for any contact $(2 n+2)$-harmonic map $\phi: \mathbb{H}_{n} \rightarrow S^{2 m-1}$ satisfying (96) for some $0<c<1$ and some $0<\delta<1$

$$
\begin{aligned}
& \left|\int_{\mathbb{B}}\left[X^{*} \cdot\left(\phi_{B} E_{A, B}\right)\right] \psi d v\right| \\
& \quad \leq C\|X \psi\|_{L^{D}(\mathbb{B})}\left\{\|X \phi\|_{L^{D}(100 \mathbb{B})}^{D}+\|X \phi\|_{L^{D}(100 \mathbb{B})}^{(1-\epsilon) D}\right\}
\end{aligned}
$$

for some constant $C=C\left(U_{1}, n, R_{0}\right)>0$, where $\epsilon=(1-\delta) / D$ and $D=2 n+2$.

This is similar to Lemma 3.2 (the duality inequality) in [7, page 354] and will be proved later on in this section.

Let $U_{1} \subset \subset U$ and $R_{0}>0$ as in Lemma 17. Also let $x \in U_{1}$ and $0<r<R_{0}$ and set $\mathbb{B}=B_{X}(x, r)$ and $2 \mathbb{B}=B_{X}(x, 2 r)$. Let $\psi \in C_{0}^{\infty}(U)$ be a test function such that $0 \leq \psi \leq 1, \psi=1$ on $\mathbb{B}, \psi=0$ on $U \backslash 2 \mathbb{B}$, and $|X \psi| \leq C / r$ for some constant $C>0$. Next let us set

$$
\psi_{A}=\left[\phi_{A}-\left(\phi_{A}\right)_{2 \mathbb{B}}\right] \psi
$$

Throughout if $(X, \mu)$ is a measurable space and $A \subset X$ a measurable set with $\mu(A)>0$, we adopt the notation $u_{A}=$ $(1 / \mu(A)) \int_{A} u d \mu$. Let us take the dot product of (79) with $X^{*}$, multiply the resulting equation by $\psi_{A}$, integrate over $2 \mathbb{B}$, and sum over $A$

$$
\begin{aligned}
\sum_{A=1}^{2 m} \int_{2 \mathbb{B}}\left(X^{*} \cdot V_{A}\right) \psi_{A} d v \\
=\sum_{A, B=1}^{2 m} \int_{2 \mathbb{B}}\left[X^{*} \cdot\left(\phi_{B} E_{A, B}\right)\right] \psi_{A} d v .
\end{aligned}
$$

The first line of (85) may be computed as follows:

$$
\begin{aligned}
\int_{2 \mathbb{B}} & \left(X^{*} \cdot V_{A}\right) \psi_{A} d v \\
= & \int_{2 \mathbb{B}} V_{A} \cdot X\left(\psi_{A}\right) d v \\
= & \int_{2 \mathbb{B}} V_{A} \cdot\left\{X(\psi)\left[\phi_{A}-\left(\phi_{A}\right)_{2 \mathbb{B}}\right]+\psi X\left(\phi_{A}\right)\right\} d v
\end{aligned}
$$

and summed over $A$

$$
\begin{aligned}
& \sum_{A} V_{A} \cdot X\left(\phi_{A}\right) \\
& =\sum_{a}\left\{V_{\alpha, a} X_{a}\left(\psi^{\alpha}\right)+V_{m+\alpha, a} X_{a}\left(\phi^{m+\alpha}\right)\right\} \\
& =Q(\phi)^{(p-2) / 2}\left\{|X \phi|^{2}-\sum_{a}\left(L_{a} \phi\right)^{2}\right\} \\
& =Q(\phi)^{p / 2}
\end{aligned}
$$


by the very definition of $V_{A}$ (cf. Lemma 22) and by (67). Thus (85) becomes

$$
\begin{aligned}
\int_{2 \mathbb{B}} \psi Q(\phi)^{p / 2} d v+\sum_{A} \int_{2 \mathbb{B}}\left[\phi_{A}-\left(\phi_{A}\right)_{2 \mathbb{B}}\right] V_{A} \cdot X(\psi) d v \\
=\sum_{A, B} \int_{2 \mathbb{B}}\left[X^{*} \cdot\left(\phi_{B} E_{A, B}\right)\right] \psi_{A} d v .
\end{aligned}
$$

For simplicity let $I_{A, B}=\int_{2 \mathbb{B}}\left[X^{*} \cdot\left(\phi_{B} E_{A, B}\right)\right] \psi_{A} d v$ and $C_{0}=$ $\sum_{A, B}\left|I_{A, B}\right|$. Using (88), we may perform the estimates

$$
\begin{aligned}
\int_{\mathbb{B}} Q(\phi)^{p / 2} d v & \leq \int_{2 \mathbb{B}} \psi Q(\phi)^{p / 2} d v \\
& \leq C_{0}+\sum_{A} \int_{2 \mathbb{B}}\left|\phi_{A}-\left(\phi_{A}\right)_{2 \mathbb{B}}\right|\left|V_{A}\right||X \psi| d v
\end{aligned}
$$

Lemma 18. Let one set $|L \phi|^{2}=\sum_{a=1}^{2 n}\left|L_{a} \phi\right|^{2}$. Then $|L X| \leq$ $\sqrt{2}|X \phi|$ a.e. in $U$ and consequently

$$
\left|V_{A}\right| \leq \sqrt{6} Q(\phi)^{(p-2) / 2}|X \phi|
$$

a.e. in $U$, for any $1 \leq A \leq 2 m$.

The inequalities in Lemma 18 follow easily from $\left|\phi_{A}\right| \leq 1$ and $\left|X \phi_{A}\right| \leq|X \phi|$. Using (90), we may write (89) as

$$
\begin{aligned}
& \int_{\mathbb{B}} Q(\phi)^{p / 2} d v \\
& \quad \leq C_{0}+\sqrt{6} \sum_{A} \int_{2 \mathbb{B}} Q(\phi)^{(p-2) / p}\left|\phi_{A}-\left(\phi_{A}\right)_{2 \mathbb{B}}\right||X \phi||X \psi| d v .
\end{aligned}
$$

In the following estimates $C$ denotes some positive constant, not necessarily the same in all formulae. By Hölder's inequality

$$
\begin{aligned}
& \int_{2 \mathbb{B}} Q(\phi)^{(p-2) / 2}\left|\phi_{A}-\left(\phi_{A}\right)_{2 \mathbb{B}}\right||X \phi||X \psi| d v \\
& \leq\left(\int_{2 \mathbb{B}}\left|\phi_{A}-\left(\phi_{A}\right)_{2 \mathbb{B}}\right|^{p} d v\right)^{1 / p} \\
& \quad \times\left(\int_{2 \mathbb{B} \backslash \mathbb{B}}\left(Q(\phi)^{(p-2) / 2}|X \phi||X \psi|\right)^{p /(p-1)} d v\right)^{(p-1) / p} \\
& \leq C\left(\int_{2 \mathbb{B}}\left|X \phi_{A}\right|^{p} d v\right)^{1 / p} \\
& \quad \times\left(\int_{2 \mathbb{B} \backslash \mathbb{B}} Q(\phi)^{p(p-2) / 2(p-1)}|X \phi|^{p /(p-1)}\right)^{(p-1) / p}
\end{aligned}
$$

by the Poincaré inequality

$$
\left(\int_{2 \mathbb{B}}\left|\phi_{A}-\left(\phi_{A}\right)_{2 \mathbb{B}}\right|^{p} d v\right)^{1 / p} \leq \operatorname{Cr}\left(\int_{2 \mathbb{B}}\left|X \phi_{A}\right|^{p} d v\right)^{1 / p}
$$

and by $|X \psi| \leq C / r$. Let us observe that $Q(\phi) \leq|X \phi|^{2}$ yields

$$
\begin{gathered}
\left(\int_{2 \mathbb{B} \backslash \mathbb{B}} Q(\phi)^{p(p-2) / 2(p-1)}|X \phi|^{p /(p-1)}\right)^{(p-1) / p} \\
\leq\left(\int_{2 \mathbb{B} \backslash \mathbb{B}}|X \phi|^{p} d v\right)^{(p-1) / p} .
\end{gathered}
$$

Hence (by (91))

$$
\begin{aligned}
\int_{\mathbb{B}} Q(\phi)^{p / 2} d v \leq & C_{0}+C\left(\int_{2 \mathbb{B}}|X \phi|^{p} d v\right)^{1 / p} \\
& \times\left(\int_{2 \mathbb{B} \backslash \mathbb{B}}|X \phi|^{p} d v\right)^{(p-1) / p} .
\end{aligned}
$$

Let us set $I_{p}(r)=\int_{B_{X}(x, r)}|X \phi|^{p} d v$. Also let us restrict our considerations to maps $\phi: \mathbb{H}_{n} \rightarrow S^{2 m-1}$ for which one may control $Q(\phi)$ from below. We adopt the following.

Definition 19. A map $\phi: \mathbb{H}_{n} \rightarrow S^{2 m-1}$ is said to be close to a horizontal map if there exist constants $0<c<1$ and $0<\delta<$ 1 such that

$$
\begin{array}{ll}
|L \phi| \leq c|X \phi|^{\delta} & \text { a.e. in }\left\{x \in \mathbb{H}_{n}:|X \phi|(x) \geq 1\right\} \\
|L \phi| \leq c|X \phi| & \text { a.e. in }\left\{x \in \mathbb{H}_{n}:|X \phi|(x)<1\right\}
\end{array}
$$

If $\phi: \mathbb{W}_{n} \rightarrow S^{2 m-1}$ is close to horizontal, then (by (96))

$$
Q(\phi) \geq a|X \phi|^{2}, \quad a=1-c^{2}>0
$$

Our main result in this section is the following.

Theorem 20. Let $U \subset \mathbb{H}_{n}$ be a bounded domain in the Heisenberg group and $\bar{Z}_{\alpha}=\partial / \partial \bar{z}^{\alpha}-i z^{\alpha} \partial / \partial t, 1 \leq \alpha \leq n$, the Lewy operators. Let $X=\left\{Z_{\alpha}+\bar{Z}_{\alpha}, i\left(Z_{\alpha}-\bar{Z}_{\alpha}\right): 1 \leq \alpha \leq n\right\}$ and $U_{1} \subset \subset U$. Let $\phi \in W_{X}^{1,2 n+2}\left(U, S^{2 m-1}\right)$ be a map obeying to (96) for some $0<c<1$ and $0<\delta<1$. If $\phi: U \rightarrow S^{2 m-1}$ is a weak contact $(2 n+2)$-harmonic map, then there exist constants $r_{0}>0, C>0$ and $0<\gamma<1$ such that

$$
\int_{B_{X}(x, r)}|X \phi|^{2 n+2} d v \leq C r^{\gamma}
$$

for any $x \in U_{1}$ and any $0<r \leq r_{0}$.

As a consequence of Theorem 20 (by applying a version of the Dirichlet growth theorem due to Macias and Segovia [15]).

Corollary 21. Let $U \subset \mathbb{M}_{n}$ be a bounded domain. Any weak contact $(2 n+2)$-harmonic map $\phi: U \rightarrow S^{2 m-1}$ satisfying (96) is locally Hölder continuous.

To prove Theorem 20, we use a hole filling technique essentially due to Widman [32], (cf. also Bensoussan et al. [31, 
page 38-40]). By (95) with $p=D=2 n+2$ and Lemma 17 with $\psi=\psi_{A}$, we have

$$
\begin{aligned}
\int_{\mathbb{B}} Q(\phi)^{D / 2} d v & \\
\leq C & \left\{I_{D}(2 r)^{1 / D}\left(I_{D}(2 r)-I_{D}(r)\right)^{(D-1) / D}\right. \\
& +\left[I_{D}(200 r)+I_{D /(D+1)}(200 r)^{1 /(D+1)}\right] \\
& \left.\times \sum_{A=1}^{2 m}\left\|X \psi_{A}\right\|_{L^{D}(2 \mathbb{B})}\right\} .
\end{aligned}
$$

On the other hand, by the very definition of $\psi_{A}$, we may use the Poincaré inequality to estimate

$$
\begin{aligned}
\sum_{A=1}^{2 m} \| & X \psi_{A} \|_{L^{D}(2 \mathbb{B})} \\
\leq & \sum_{a}\left\{\left\|(X \psi)\left[\phi_{A}-\left(\phi_{A}\right)_{2 \mathbb{B}}\right]\right\|_{L^{D}(2 \mathbb{B})}+\left\|\psi X \phi_{A}\right\|_{L^{D}(2 \mathbb{B})}\right\} \\
= & \sum_{A}\left(\int_{2 \mathbb{B}}|X \psi|^{D}\left|\phi_{A}-\left(\phi_{A}\right)_{2 \mathbb{B}}\right|^{D} d v\right)^{1 / D} \\
& +\sum_{A}\left(\int_{2 \mathbb{B}}|\psi|^{D}\left|X \phi_{A}\right|^{D} d v\right)^{1 / D} \\
\leq & \frac{C}{r} \sum_{A}\left(\int_{2 \mathbb{B}}\left|\phi_{A}-\left(\phi_{A}\right)_{2 \mathbb{B}}\right|^{D} d v\right)^{1 / D} \\
& +\sum_{A}\left(\int_{2 \mathbb{B}}\left|X \phi_{A}\right|^{D} d v\right)^{1 / D},
\end{aligned}
$$

that is,

$$
\sum_{A=1}^{2 m}\left\|X \psi_{A}\right\|_{L^{D}(2 \mathbb{B})} \leq C I_{D}(2 r)^{1 / D} .
$$

Using (97) and (101), the inequality (120) yields

$$
\begin{aligned}
& a I_{D}(r) \\
& \leq C\left\{I_{D}(2 r)^{1 / D}\left(I_{D}(2 r)-I_{D}(r)\right)^{(D-1) / D}\right. \\
& \left.+\left[I_{D}(200 r)+I_{D}(200 r)^{1-\epsilon}\right] I_{D}(2 r)^{1 / D}\right\} .
\end{aligned}
$$

By the Vitali absolute continuity of the integral $I_{D}(200 r)$, there is $r_{0}^{\prime}>0$ such that $I_{D}(200 r)<1$ for any $0<r \leq r_{0}^{\prime}$. As a consequence of (102) we may establish the following.

Lemma 22. There exist $0<r_{0} \leq r_{0}^{\prime}$ and $1 / 2 \leq \lambda<1$ such that

$$
I_{D}(r) \leq \lambda I_{D}(200 r)^{1-\epsilon}
$$

for any $0<r \leq r_{0}$.
Proof. The proof is by contradiction. Let us assume that for any $0<r_{0} \leq r_{0}^{\prime}$ and any $1 / 2 \leq \lambda<1$, there is $0<r \leq r_{0}$ such that $I(r)>\lambda I(200 r)^{1-\epsilon}$, where $I$ is short for $I_{D}$. Note that $I(200 r) \leq I(200 r)^{1-\epsilon}$. Then (by (102))

$$
\begin{aligned}
\lambda I(200 r)^{1-\epsilon}< & \text { I }(r) \\
\leq & C\left\{I(200 r)(1-\lambda)^{(D-1) / D}\right. \\
& \left.\quad+\left[I(200 r)+I(200 r)^{1-\epsilon}\right] I(2 r)^{1 / D}\right\} \\
& \leq C I(200 r)^{1-\epsilon}\left\{(1-\lambda)^{(D-1) / D}+I(2 r)^{1 / D}\right\} .
\end{aligned}
$$

Therefore

$$
\frac{1}{2} \leq \lambda<C\left\{(1-\lambda)^{(D-1) / D}+I(2 r)^{1 / D}\right\} .
$$

The inequality (105) leads to

$$
\left(\frac{1}{2 C}\right)^{D} \leq \int_{2 \mathbb{B}}|X \phi|^{D} d \nu .
$$

Indeed, by the contradiction assumption, we may pick a sequence $\lambda_{j} \in[1 / 2,1)$ such that $\lambda_{j} \rightarrow 1$ as $j \rightarrow \infty$ and consider the corresponding radii $0<r_{j} \leq r_{0}$. By passing to a subsequence, if necessary, one may assume that $\lim _{j \rightarrow \infty} r_{j}=$ $r_{\infty}$ for some $r_{\infty} \in\left[0, r_{0}\right]$. Let $j \rightarrow \infty$ in

$$
\frac{1}{2} \leq \lambda_{j}<C\left\{\left(1-\lambda_{j}\right)^{(D-1) / D}+I\left(2 r_{j}\right)^{1 / D}\right\}
$$

and use the absolute continuity of the integral. Then either $r_{\infty}>0$ (yielding (106)) or $r_{\infty}=0$ and then $1 / 2 \leq 0$, a contradiction. Finally (106) may be exploited as follows. Let $r_{0}=1 / k$. By the contradiction assumption there is $0<r \leq$ $1 / k$ such that (by (106))

$$
\left(\frac{1}{2 C}\right)^{D} \leq I(2 r) \leq \int_{B_{X}(x, 2 / k)}|X \phi|^{D} d v
$$

and the last integral tends to 0 as $k \rightarrow \infty$, a contradiction. Lemma 22 is proved.

Now we may prove the Caccioppoli type estimate (98). Let $\tau=1 / 200$ so that (103) may be written as

$$
I_{D}(\tau r) \leq \lambda I_{D}(r)^{1-\epsilon}
$$

Then (by (109) and induction over $m$ )

$$
I_{D}\left(\tau^{m} r\right) \leq \lambda^{\left[1-(1-\epsilon)^{m}\right] / \epsilon} I_{D}(r)^{(1-\epsilon)^{m}}
$$

for any $m \in \mathbb{Z}, m \geq 1$. Let us consider the family of intervals $\left\{\left(\tau^{m}, \tau^{m-1}\right]: m \in \mathbb{Z}, m \geq 1\right\}$. It is a cover of $(0,1]$, hence for each $0<r \leq r_{0}$ there is $m \in \mathbb{Z}, m \geq 1$, such that $\tau^{m}<r / r_{0} \leq$ $\tau^{m-1}$. Now the inequality $r \leq \tau^{m-1} r_{0}$ implies (by (110))

$$
I_{D}(r) \leq I_{D}\left(\tau^{m-1} r_{0}\right) \leq \lambda^{\left[1-(1-\epsilon)^{m-1}\right] / \epsilon} I_{D}\left(r_{0}\right)^{(1-\epsilon)^{m-1}}
$$


On the other hand let us set $\gamma=(\log \lambda) /(\log \tau)$ (so that $0<$ $\gamma<1$ ) and observe that the inequality $r / r_{0} \geq \tau^{m}$ implies

$$
\left(\frac{r}{r_{0}}\right)^{\gamma}>\tau^{m \gamma}=\tau^{\left(\log \lambda^{m}\right) /(\log \tau)}=\lambda^{m}
$$

that is, $\lambda^{m}<\left(r / r_{0}\right)^{\gamma}$. One may choose $r_{0}>0$ from the very beginning such that $I_{D}\left(r_{0}\right)<\lambda$ for any $x \in U_{1}$. Note that $0<\epsilon \leq 1 / 2$ (by the very definition of $\epsilon$ ). Then $[1-(1-$ $\left.\epsilon)^{m}\right] / \epsilon=\sum_{j=0}^{m-1}(1-\epsilon)^{j} \geq 1+(m-1)(1 / 2)=(m+1) / 2$, hence $\lambda^{\left[1-(1-\epsilon)^{m}\right] / \epsilon} \leq C r^{\gamma / 2}$, where $C=r_{0}^{-\gamma / 2} \sqrt{\lambda}$. Theorem 20 is proved.

It remains that we prove Lemma 17. It suffices to prove the inequality (83) for any $\psi \in C_{0}^{\infty}(\mathbb{B})$. Let us consider

$$
w(x)=\left(\frac{1}{4 a_{0}}\right)|x|^{-2 n}, \quad x \in \mathbb{H}_{n},
$$

where $a_{0}=\left(2^{2-2 n} \pi^{n+1} / \Gamma(n / 2)\right)^{2}$ and $|x|=\left(|z|^{4}+t^{2}\right)^{1 / 4}$ is the Heisenberg norm of $x=(z, t)$. By a classical result of Folland, [33], $G(x, y)=w\left(x y^{-1}\right)$ is a fundamental solution for the Hörmander operator $\sum_{a=1}^{2 n} X_{a}^{2}$. In particular for any bounded domain $U \subset \mathbb{H}_{n}$ one has the representation formula

$$
u(x)=\int_{U} X_{y} G(y, x) \cdot X u(y) d v(y)
$$

for any $u \in C_{0}^{\infty}(U)$ and any $x \in U$. By a result of Citti et al., [34], we may consider a smooth cut-off function $0 \leq$ $\psi_{0} \leq 1$ such that $\psi_{0}=1$ on $2 \mathbb{B}, \psi_{0}=0$ on $U \backslash 4 \mathbb{B}$, and $\left|X \psi_{0}\right| \leq C / \operatorname{diam}(\mathbb{B})$ (the diameter is meant with respect to the Carnot-Carathéodory metric on $\mathbb{H}_{n}$ ). Using (114) for $u=\psi$, one may write

$$
\begin{aligned}
\int_{\mathbb{B}} & {\left[X^{*} \cdot\left(\phi_{B} E_{A, B}\right)\right] \psi d v } \\
= & \int_{\mathbb{B}} X^{*} \cdot\left(\phi_{B} E_{A, B}\right)(x) \psi(x) \psi_{0}(x) d v(x) \\
= & \int_{\mathbb{B}} d v(x)\left[X^{*} \cdot\left(\phi_{B} E_{A, B}\right)\right](x) \psi_{0}(x) \\
& \times \int_{\mathbb{B}} X_{y} G(y, x) \cdot X \psi(y) d v(y) \\
= & \int_{\mathbb{B}} \mathscr{A}_{A, B} \cdot(X \psi) d v,
\end{aligned}
$$

where we have set

$$
\mathscr{A}_{A, B}(y)=\int_{\mathbb{B}}\left[X^{*} \cdot\left(\phi_{B} E_{A, B}\right)\right](x) \psi_{0}(x) X_{y} G(y, x) d v(x) \text {. }
$$

We wish to prove an estimate on $|\mathscr{A}(y)|$, where $\mathscr{A}=\mathscr{A}_{A, B}$ for simplicity. As it is well known, $\left|B_{X}(x, r)\right|=C r^{2 n+2}$ for some constant $C>0$ and any $x \in \mathbb{H}_{n}$ and $r>0$. Here $|A|$ denotes the Lebesgue measure of the set $A$. In particular the Lebesgue measure on $\left(\mathbb{U}_{n}, d_{X}\right)$ has the doubling property. Thus we may apply a result by Macias and Segovia, [15], to pick a Whitney decomposition of $U_{y}=U \backslash\{y\}$. Precisely let $y \in \mathbb{B}$, and given $x \in U_{y}$, let us set $r_{x}=d_{X}\left(x, \mathbb{H}_{n} \backslash U_{y}\right) / 1000$. Next let us choose among $\left\{B_{X}\left(x, r_{x}\right)\right\}_{x \in U_{y}}$ a maximal family of mutually disjoint balls $\left\{B_{X}\left(x_{\alpha}, r_{\alpha}\right)\right\}_{\alpha \in I}$. Then $U_{y}=\bigcup_{\alpha \in I} B_{X}\left(x_{\alpha}, 3 r_{\alpha}\right)$ (the Whitney decomposition of $\left.U_{y}\right)$ and there is $N \geq 1$ such that each $x \in U$ belongs to at most $N$ balls $B_{X}\left(x_{\alpha}, 6 r_{\alpha}\right)$. Moreover, again by a result in [15], we may associate a partition of unity to the Whitney decomposition of $U_{y}$; that is, we may consider a family of smooth functions $\left\{\theta_{\alpha}\right\}_{\alpha \in I}$ such that $0 \leq \theta_{\alpha} \leq 1$, $\sum_{\alpha \in I} \theta_{\alpha}=1$ on $U_{y}, \operatorname{Supp}\left(\theta_{\alpha}\right) \subset \mathbb{B}_{\alpha}=B_{X}\left(x_{\alpha}, 6 r_{\alpha}\right)$, and $\left|X \theta_{\alpha}\right| \leq C / r_{\alpha}$. The bounds on the gradients actually follow from the work by Citti et al., [34], quoted above. Then

$$
\begin{gathered}
\mathscr{A}_{a}(y) \\
=\sum_{\alpha \in I} \int_{\mathbb{B}_{\alpha}}\left[X^{*} \cdot\left(\phi_{B} E_{A, B}\right)\right](x) \psi_{0}(x) \\
\quad \times \theta_{\alpha}(x) X_{a, y} G(y, x) d v(x) \\
=\sum_{\alpha \in I} \int_{\mathbb{B}_{\alpha}}\left[X^{*} \cdot\left(\phi_{B}-\left(\phi_{B}\right)_{\mathbb{B}_{\alpha}}\right) E_{A, B}\right](x) \psi_{0}(x) \\
\quad \times \theta_{\alpha}(x) X_{a, y} G(y, x) d v(x) \\
+\sum_{\alpha \in I}\left(\phi_{B}\right)_{\mathbb{B}_{\alpha}} \int_{\mathbb{B}_{\alpha}}\left(X^{*} \cdot E_{A, B}\right)(x) \psi_{0}(x) \\
\times \theta_{\alpha}(x) X_{a, y} G(y, x) d v(x) \\
=\mathscr{A}_{a}^{\prime}(y)+\mathscr{A}_{a}^{\prime \prime}(y) .
\end{gathered}
$$

The presence of term $\mathscr{A}_{a}^{\prime \prime}(y)$ represents of course the main difference with respect to the proof of the so called duality inequality in [7] (there $X^{*} \cdot E_{A, B}=0$ ). Integrating by parts,

$$
\begin{aligned}
& \mathscr{A}_{a}^{\prime}(y) \\
& =\sum_{\alpha \in I} \int_{\mathbb{B}_{\alpha}}\left(\phi_{B}(x)-\left(\phi_{B}\right)_{\mathbb{B}_{\alpha}}\right) \\
& \quad \times E_{A, B}(x) \cdot X_{x}\left[\psi_{0}(x) \theta_{\alpha}(x) X_{a, y} G(y, x)\right] d v(x) .
\end{aligned}
$$

Due to the explicit form of the fundamental solution $G(x, y)$, one may easily check that

$$
\begin{gathered}
\left|X_{a} G(x, y)\right| \leq C d_{X}(x, y)^{-2 n-1}, \\
\left|X_{a} X_{b} G(x, y)\right| \leq C d_{X}(x, y)^{-2 n-2},
\end{gathered}
$$

for any $x, y \in U$. Here it is irrelevant whether differentiation is performed in $x$ or $y$. Estimates of the sort in the case of an arbitrary Hörmander system of vector fields have been obtained by Sánchez-Calle [35]. Estimates on $G(x, y)$ itself are available, yet only estimates on the derivatives are needed for the following calculations. Using (119)-(120) and

$$
\begin{gathered}
\left|X \psi_{0}(x)\right| \leq C d_{X}(x, y)^{-1}, \\
\left|\theta_{\alpha}(x)\right| \leq C d_{X}(x, y)^{-1}, \quad \alpha \in I,
\end{gathered}
$$


one has

$$
\left|X_{b, x}\left[\psi_{0}(x) \theta_{\alpha}(x) X_{a, y} G(y, x)\right]\right| \leq C d_{X}(x, y)^{-2 n-2},
$$

hence

$$
\left|\mathscr{A}_{\alpha}^{\prime}(y)\right| \leq C \sum_{\alpha \in I} \int_{\Gamma_{\alpha}} \frac{\left|\phi_{B}(x)-\left(\phi_{B}\right)_{\mathbb{B}_{\alpha}}\right|\left|E_{A, B}(x)\right|}{d_{X}(x, y)^{2 n+2}} d v(x),
$$

where $\Gamma_{\alpha}=\operatorname{Supp}\left(\theta_{\alpha}\right)$. Let $x \in \mathbb{B}_{\alpha}=B_{X}\left(x_{\alpha}, 6 r_{\alpha}\right)$. As $y \in$ $\mathbb{U}_{n} \backslash U_{y}$, the very definition of $r_{\alpha}$ yields $d_{X}\left(y, x_{\alpha}\right) \geq 1000 r_{\alpha}$; hence

$$
\begin{aligned}
1000 r_{\alpha} & \leq d_{X}\left(y, x_{\alpha}\right) \\
& \leq d_{X}(y, x)+d_{X}\left(x, x_{\alpha}\right) \\
& \leq d_{X}(x, y)+6 r_{\alpha}
\end{aligned}
$$

and in particular $6 r_{\alpha} \leq d_{X}(x, y)$. Thus $\left|\mathbb{B}_{\alpha}\right|=C r_{\alpha}^{2 n+2} \leq$ $C^{\prime} d_{X}(x, y)^{2 n+2}$, where $C^{\prime}=C 6^{-2 n-2}$; hence there is a constant $C>0$ such that

$$
d_{X}(x, y)^{2 n+2} \geq C\left|\mathbb{B}_{\alpha}\right|, \quad x \in \mathbb{B}_{\alpha} .
$$

Let us set $J=\left\{\alpha \in I: \Gamma_{\alpha} \cap 4 \mathbb{B} \neq \emptyset\right\}$. Let us apply (123) and (125) and Hölder's inequality to perform the estimates

$$
\begin{aligned}
\left|\mathscr{A}_{a}^{\prime}(y)\right| \leq & C \sum_{\alpha \in J} \frac{1}{\left|\mathbb{B}_{\alpha}\right|} \int_{\mathbb{B}_{\alpha}}\left|\phi_{B}(x)-\left(\phi_{B}\right)_{\mathbb{B}_{\alpha}}\right|\left|E_{A, B}(x)\right| d v(x) \\
\leq & C \sum_{\alpha \in J}\left(\frac{1}{\left|\mathbb{B}_{\alpha}\right|} \int_{\mathbb{B}_{\alpha}}\left|\phi_{B}-\left(\phi_{B}\right)_{\mathbb{B}_{\alpha}}\right|^{D^{2}} d v\right)^{1 / D^{2}} \\
& \times\left(\frac{1}{\left|\mathbb{B}_{\alpha}\right|} \int_{\mathbb{B}_{\alpha}}\left|E_{A, B}\right|^{D^{2} /\left(D^{2}-1\right)} d v\right)^{\left(D^{2}-1\right) / D^{2}},
\end{aligned}
$$

where we have set $D=2 n+2$ for simplicity. By (90) in Lemma 18 and $Q \leq|X \phi|^{2}$, one has $\left|E_{A, B}\right| \leq 2 \sqrt{6}|X \phi|^{D-1}$; hence

$$
\begin{aligned}
& \left(\frac{1}{\left|\mathbb{B}_{\alpha}\right|} \int_{\mathbb{B}_{\alpha}}\left|E_{A, B}\right|^{D^{2} /\left(D^{2}-1\right)} d v\right)^{\left(D^{2}-1\right) / D^{2}} \\
& \quad \leq C\left(\frac{1}{\left|\mathbb{B}_{\alpha}\right|}|X \phi|^{D^{2} /(D+1)} d v\right)^{\left(D^{2}-1\right) / D^{2}} .
\end{aligned}
$$

At this point we need to apply a version of the Sobolev inequality due to Franchi et al. [36]. Precisely, for any
$1 \leq p<2 n+2$ there is a constant $C>0$ such that for any ball $B_{X}(x, r)$ with $x \in U$ and $0<r \leq \operatorname{diam}(U)$

$$
\begin{array}{r}
\left(\frac{1}{\left|B_{X}(x, r)\right|} \int_{B_{X}(x, r)}\left|u-u_{B_{X}(x, r)}\right|^{p^{*}} d v\right)^{1 / p^{*}} \\
\leq \operatorname{Cr}\left(\frac{1}{\left|B_{X}(x, r)\right|} \int_{B_{X}(x, r)}|X u|^{p} d v\right)^{1 / p}, \\
p^{*}=\frac{2(n+1) p}{2 n+2-p} .
\end{array}
$$

By the assumption in Theorem 20 one has $X_{a} \phi_{B} \in L^{2 n+2}(U)$; hence $X_{a} \phi_{B} \in L^{v}(U)$ for any $0<v \leq 2 n+2$. Therefore (by the Sobolev inequality above)

$$
\begin{aligned}
& \left(\frac{1}{\left|\mathbb{B}_{\alpha}\right|}\left|\phi_{B}-\left(\phi_{B}\right)_{\mathbb{B}_{\alpha}}\right|^{D^{2}} d v\right)^{1 / D^{2}} \\
& \quad \leq C r_{\alpha}\left(\frac{1}{\left|\mathbb{B}_{\alpha}\right|} \int_{\mathbb{B}_{\alpha}}|X \phi|^{d^{2} /(D+1)} d v\right)^{(D+1) / D^{2}} .
\end{aligned}
$$

Collecting the information in (127) and (129),

$$
\left|\mathscr{A}_{a}^{\prime}(y)\right| \leq C \sum_{\alpha \in J} r_{\alpha}\left(\frac{1}{\left|\mathbb{B}_{\alpha}\right|} \int_{\mathbb{B}_{\alpha}}|X \phi|^{D^{2} /(D+1)} d v\right)^{(D+1) / D} .
$$

In the sequel we write briefly $a \approx b$ whenever $a / C \leq b \leq$ $\mathrm{Ca}$ for some constant $C \geq 1$. Let $\alpha \in J$. If there is $k \in \mathbb{Z}$ such that $x_{\alpha} \in B_{X}\left(y, 2^{k-1}\right) \backslash B_{X}\left(y, 2^{k-2}\right)$, then $r_{\alpha} \approx 2^{k}$ and $\mathbb{B}_{\alpha} \subset B_{X}\left(y, 2^{k}\right)$ (our arguments follow closely those in [7, page 356$])$. Moreover

$$
\frac{\left|\mathbb{B}_{\alpha}\right|}{\left|B_{X}\left(y, 2^{k}\right)\right|}=\left(\frac{6 r_{\alpha}}{2^{k}}\right)^{2 n+2},
$$

hence $\left|\mathbb{B}_{\alpha}\right| \approx\left|B_{X}\left(y, 2^{k}\right)\right|$. Consequently

$$
\begin{aligned}
& r_{\alpha}\left(\frac{1}{\left|\mathbb{B}_{\alpha}\right|} \int_{\mathbb{B}_{\alpha}}|X \phi|^{D^{2} /(D+1)} d v\right)^{(D+1) / D} \\
& \quad \leq C 2^{k}\left(\frac{1}{\left|B_{X}\left(y, 2^{k}\right)\right|} \int_{B_{X}\left(y, 2^{k}\right)}|X \phi|^{D^{2} /(D+1)} d v\right)^{(D+1) / D} .
\end{aligned}
$$

Also $\left\{\alpha \in J: x_{\alpha} \in B_{X}\left(y, 2^{k-1}\right) \backslash B_{X}\left(x_{\alpha}, 2^{k-2}\right)\right\}=\emptyset$ whenever $2^{k-2} \geq \operatorname{diam}(8 \mathbb{B})$ and the estimate (130) may be written as

$\left|\mathscr{A}_{a}^{\prime}(y)\right|$

$\leq C \sum_{2^{k} \leq 4 \operatorname{diam}(8 \mathbb{B})} 2^{k}\left(\frac{1}{\left|B_{X}\left(y, 2^{k}\right)\right|} \int_{B_{X}\left(y, 2^{k}\right)}|X \phi|^{D^{2} /(D+1)} d v\right)^{(D+1) / D}$.

Next we shall express the estimate on $\left|\mathscr{A}_{a}^{\prime}(y)\right|$ in terms of Riesz potentials and then use the general estimates on $L^{p}$ 
norms of Riesz potentials as obtained by Hàjlasz and Koskela [37]. To recall the needed result, let $(X, \rho)$ be a metric space endowed with a Borel measure $\mu$ such that $\mu(B)>0$ for any ball $B \subset X$. Let $A \subset X$ be a bounded open set and let us consider the numbers $q>0, \sigma \geq 1$, and $h>0$.

Definition 23. An (abstract) Riesz potential operator $J_{h, q}^{\sigma, A}$ is given by

$$
\begin{aligned}
& \left(J_{h, q}^{\sigma, A} g\right)(x) \\
& =\sum_{2^{k} \leq 2 \sigma \operatorname{diam}(A)} 2^{k h}\left(\frac{1}{\left|B\left(x, 2^{k}\right)\right|} \int_{B\left(x, 2^{k}\right)}|g(z)|^{q} d \mu(z)\right)^{1 / q} .
\end{aligned}
$$

The estimate (133) implies

$$
\left|\mathscr{A}_{a}^{\prime}(y)\right| \leq C\left(J_{1, q}^{2,8 \mathbb{B}}|X \phi|\right)(y), \quad q=\frac{D}{D+1} .
$$

The needed result in [37] holds for an arbitrary metric space $(X, \rho)$ endowed with a Borel measure $\mu$ such that $\mu(B)>0$ for any ball $B \subset X$. Let $A \subset X$ be a bounded open set such that $\mu$ is doubling on

$$
V=\{x \in X: \operatorname{dist}(x, A)<2 \sigma \operatorname{diam}(A)\} .
$$

Let us assume that there are constants $b>0$ and $D>0$ such that

$$
\mu(B(x, R)) \geq b\left(\frac{R}{\operatorname{diam}(A)}\right)^{D} \mu(A)
$$

for any $x \in A$ and any $0<R \leq 2 \sigma \operatorname{diam}(A)$. Moreover let $h>0$ and $0<q \leq s<D / h$. Then (cf. [37])

$$
\left\|J_{h, q}^{\sigma, A} g\right\|_{L^{s^{*}}(A, \mu)} \leq C\left(\frac{\operatorname{diam}(A)}{\mu(A)^{1 / D}}\right)^{h}\|g\|_{L^{s}(V, \mu)},
$$

where $s^{*}=s D /(D-h s)$ and the constant $C>0$ depends only on $h, \sigma, q, s, b, D$, and the doubling constant. Then (by Hölder's inequality with $1 /(2 n+2)+1 / D^{\prime}=1$, resp., with $1 / \mu+1 / \mu^{\prime}=1$ )

$$
\begin{aligned}
& \left|\int_{\mathbb{B}}\left[X^{*} \cdot\left(\phi_{B} E_{A, B}\right)\right] \psi d v\right| \\
& \leq \sum_{a=1}^{2 n}\|X \psi\|_{L^{2 n+2}(\mathbb{B})} \\
& \quad \times\left(\int_{\mathbb{B}}\left|\mathscr{A}_{a}^{\prime}(y)\right|^{2(n+1) /(2 n+1)} d v(y)\right)^{(2 n+1) /[2(n+1)]} \\
& \quad+\sum_{a}\|X \psi\|_{L^{\mu}(\mathbb{B})}\left(\int_{\mathbb{B}}\left|\mathscr{A}_{a}^{\prime \prime}(y)\right|^{\mu^{\prime}} d v(y)\right)^{1 / \mu^{\prime}}
\end{aligned}
$$

with $1<\mu<D$ to be determined later on. At this point we need an estimate on $\left|\mathscr{A}_{a}^{\prime \prime}(y)\right|$. By (80) in Proposition 16 if $\phi: \mathbb{H}_{n} \rightarrow S^{2 m-1}$ is a contact $(2 n+2)$-harmonic map obeying to our assumptions (96), then

$$
\left|X^{*} \cdot E_{A, B}\right| \leq 2 Q(\phi)^{(D-2) / 2}|X \phi||L \phi| \leq|2 c X \phi|^{D-1+\delta}
$$

hence (by (119))

$$
\begin{aligned}
& \left|\mathscr{A}_{a}^{\prime \prime}(y)\right| \\
& \leq \sum_{\alpha \in I} \int_{\mathbb{B}_{\alpha}}\left|\left(X^{*} \cdot E_{A, B}\right)(x)\right|\left|\psi_{0}(x)\right| \\
& \quad \times\left|\theta_{\alpha}(x)\right|\left|X_{a, y} G(y, x)\right| d v(x) \\
& \leq C \sum_{\alpha \in I} \int_{\Gamma_{\alpha}} \frac{|X \phi|^{\nu}}{d_{X}(x, y)^{2 n+1}} d v(x),
\end{aligned}
$$

where $v=D-1+\delta$ and $0<v<D$. By $d_{X}(x, y)^{2 n+1} \geq$ $C\left|\mathbb{B}_{\alpha}\right| / r_{\alpha}$ for any $x \in \mathbb{B}_{\alpha}$ one obtains

$$
\begin{aligned}
\left|\mathscr{A}_{a}^{\prime \prime}(y)\right| & \leq C \sum_{\alpha \in J} r_{\alpha} \frac{1}{\left|\mathbb{B}_{\alpha}\right|} \int_{\mathbb{B}_{\alpha}}|X \phi|^{\nu} d v \\
& \leq C \sum_{\alpha \in J} \frac{r_{\alpha}}{\left|\mathbb{B}_{\alpha}\right|}\left(\int_{\mathbb{B}_{\alpha}}|X \phi|^{D}\right)^{\nu / D}\left|\mathbb{B}_{\alpha}\right|^{(D-v) / D}
\end{aligned}
$$

that is,

$$
\begin{aligned}
& \left|\mathscr{A}_{a}^{\prime \prime}(y)\right| \\
& \leq C \sum_{2^{k} \leq 4 \operatorname{diam}(8 \mathbb{B})} 2^{k}\left(\frac{1}{\left|B_{X}\left(y, 2^{k}\right)\right|} \int_{B_{X}\left(y, 2^{k}\right)}|X \phi|^{D} d v\right)^{v / D},
\end{aligned}
$$

hence

$$
\left|\mathscr{A}_{a}^{\prime \prime}(y)\right| \leq C\left(J_{1, D / \nu}^{2,8 \mathbb{B}}|X \phi|^{\nu}\right)(y) .
$$

Therefore (by (135) and (144))

$$
\begin{aligned}
& \left(\int_{\mathbb{B}}\left|\mathscr{A}_{a}^{\prime}\right|^{D /(D-1)} d v\right)^{(D-1) / D} \\
& \leq C\left\|J_{1, D /(D+1)}^{2,8 \mathbb{B}}|X \phi|^{D}\right\|_{L^{D /(D-1)}(8 \mathbb{B})} \\
& \leq C \frac{\operatorname{diam}(8 \mathbb{B})}{|8 \mathbb{B}|^{1 / D}}\left\||X \phi|^{D}\right\|_{L^{1}(V)},
\end{aligned}
$$

that is,

$$
\left(\int_{\mathbb{B}}\left|\mathscr{A}_{a}^{\prime}\right|^{D /(D-1)} d v\right)^{(D-1) / D} \leq C\|X \phi\|_{L^{D}(100 \mathbb{B})}^{D},
$$

where $V=\left\{x \in \mathbb{H}_{n}: \operatorname{dist}(x, 8 \mathbb{B}) \leq 4 \operatorname{diam}(8 \mathbb{B})\right\}$, respectively,

$$
\begin{gathered}
\left(\int_{\mathbb{B}}\left|\mathscr{A}_{a}^{\prime \prime}\right|^{\mu /(\mu-1)} d v\right)^{(\mu-1) / \mu} \\
\leq C\left\|J_{1, D / \nu}^{2,8 \mathbb{B}}|X \phi|^{\nu}\right\|_{L^{\mu / \mu-1)}(8 \mathbb{B})} \\
\leq C \frac{\operatorname{diam}(8 \mathbb{B})}{|8 \mathbb{B}|^{1 / D}}\left\||X \phi|^{\nu}\right\|_{L^{s}(V)} \\
\leq C\left\||X \phi|^{\nu}\right\|_{L^{s}(100 \mathbb{B})},
\end{gathered}
$$


where

$$
0<\frac{D}{\nu} \leq s<D, \quad \frac{\mu}{\mu-1}=s^{*}=\frac{s D}{D-s} .
$$

Therefore it must be that

$$
1<\mu \leq \frac{D}{2-\delta}, \quad s=\frac{\mu D}{(D+1) \mu-D} .
$$

On the other hand

$$
\begin{aligned}
& \left\||X \phi|^{\nu}\right\|_{L^{s}(100 \mathbb{B})} \\
& \quad=\left(\int_{100 \mathbb{B}}\left(|X \phi|^{D}\right)^{\nu \mu /[(D+1) \mu-D]} d \nu\right)^{[(D+1) \mu-D] /(\mu D)}
\end{aligned}
$$

and we may choose $\mu$ such that $\nu \mu /[(D+1) \mu-D]=1$; that is, $\mu=D /(2-\delta)$. Consequently

$$
\begin{gathered}
\frac{(D+1) \mu-D}{\mu D}=1-\epsilon, \quad \epsilon=\frac{1-\delta}{D}, \\
\left\||X \phi|^{\nu}\right\|_{L^{s}(100 \mathbb{B})}=\left(\int_{100 \mathbb{B}}|X \phi|^{D} d v\right)^{1-\epsilon}, \\
\left(\int_{\mathbb{B}}\left|\mathscr{A}_{a}^{\prime \prime}\right|^{\mu /(\mu-1)} d v\right)^{(\mu-1) / \mu} \leq C\left(\int_{100 \mathbb{B}}|X \phi|^{D} d v\right)^{1-\epsilon} .
\end{gathered}
$$

Also

$$
\begin{aligned}
\|X \psi\|_{L^{\mu}(\mathbb{B})}^{\mu}= & \int_{\mathbb{B}}|X \psi|^{\mu} d v \\
& \leq\left(\int_{\mathbb{B}}|X \psi|^{\mu(2-\delta)} d v\right)^{1 /(2-\delta)}|\mathbb{B}|^{(1-\delta) /(2-\delta)} \\
& \leq C\left(\int_{\mathbb{B}}|X \psi|^{D} d v\right)^{1 /(2-\delta)},
\end{aligned}
$$

that is, $\|X \psi\|_{L^{\mu}(\mathbb{B})} \leq C\|X \psi\|_{L^{D}(\mathbb{B})}$. Summing up (by (139) and (146) and (151)),

$$
\begin{aligned}
& \left|\int_{\mathbb{B}}\left[X^{*} \cdot\left(\phi_{B} E_{A, B}\right)\right] \psi d v\right| \\
& \quad \leq\|X \psi\|_{L^{D}(\mathbb{B})}\left\{\|X \phi\|_{L^{D}(100 \mathbb{B})}^{D}+\|X \phi\|_{L^{D}(100 \mathbb{B})}^{(1-\epsilon) D}\right\}
\end{aligned}
$$

which is (83). Lemma 17 is proved.

\section{Acknowledgments}

S. Dragomir was a visiting professor of CNRS at Laboratoire de Mathématiques Jean Leray, Université de Nantes, France (June 2008), and expresses his gratitude for the excellent work conditions there. He is also grateful to G. Citti (University of Bologna) for discussions on Example 3 in this paper.

\section{References}

[1] E. Barletta, S. Dragomir, and H. Urakawa, "Pseudoharmonic maps from nondegenerate CR manifolds to Riemannian manifolds," Indiana University Mathematics Journal, vol. 50, no. 2, pp. 719-746, 2001.
[2] E. Barletta, "Hörmander systems and harmonic morphisms," Annali della Scuola Normale Superiore di Pisa-Scienze Fisiche e Matematiche V, vol. 2, no. 2, pp. 379-394, 2003.

[3] E. Barletta, "Subelliptic F-harmonic maps," Rivista di Matematica della Università di Parma, vol. 7, no. 2, pp. 33-50, 2003.

[4] F. Hélein, Harmonic Maps, Conservation Laws and Moving Frames, vol. 150 of Cambridge Tracts in Mathematics, Cambridge University Press, Cambridge, UK, 2nd edition, 2002.

[5] J. Jost and C. J. Xu, "Subelliptic harmonic maps," Transactions of the American Mathematical Society, vol. 350, no. 11, pp. 46334649, 1998.

[6] Z.-R. Zhou, "Uniqueness of subelliptic harmonic maps," Annals of Global Analysis and Geometry, vol. 17, no. 6, pp. 581-594, 1999.

[7] P. Hajłasz and P. Strzelecki, "Subelliptic p-harmonic maps into spheres and the ghost of Hardy spaces," Mathematische Annalen, vol. 312, no. 2, pp. 341-362, 1998.

[8] C. Wang, "Subelliptic harmonic maps from Carnot groups," Calculus of Variations and Partial Differential Equations, vol. 18, no. 1, pp. 95-115, 2003.

[9] S. Dragomir and G. Tomassini, Differential Geometry and Analysis in CR Manifolds, vol. 246 of Progress in Mathematics, Birkhäuser, Berlin, Germany, 2006.

[10] S. Dragomir and R. Petit, "Contact harmonic maps," Differential Geometry and Its Applications, vol. 30, pp. 65-84, 2012.

[11] T. Ishihara, "A mapping of Riemannian manifolds which preserves harmonic functions," Kyoto Journal of Mathematics, vol. 19, no. 2, pp. 215-229, 1979.

[12] N. Garofalo and S. D. Pauls, The Bernstein Problem in the Heisenberg Group, 2002.

[13] D. E. Blair, Riemannian Geometry of Contact and Symplectic Manifolds, vol. 203 of Progress in Mathematics, Birkhäuser, Berlin, Germany, 2002.

[14] K. Yano, "On a structure defined by a tensor field $\mathrm{f}$ of type $(1,1)$ satisfying $f^{3}+f=0$," North-Holland Mathematics Studies $C$, vol. 70, pp. 251-261, 1982.

[15] R. A. Macías and C. Segovia, "A decomposition into atoms of distributions on spaces of homogeneous type," Advances in Mathematics, vol. 33, no. 3, pp. 271-309, 1979.

[16] S. Tanno, "Variational problems on contact Riemannian manifolds," Transactions of the American Mathematical Society, vol. 314, no. 1, pp. 349-379, 1989.

[17] A. Boggess, CR Manifolds and the Tangential Cauchy-Riemann Complex, Studies in Advanced Mathematics, CRC Press, London, UK, 1991.

[18] S. M. Webster, "Pseudohermitian structures on a real hypersurface," Journal of Differential Geometry, vol. 13, pp. 25-41, 1978.

[19] N. Tanaka, A Differential Geometric Study on Strongly PseudoConvex Manifolds, Kinokuniya Book Store, Kyoto, Japan, 1975.

[20] J. M. Lee, "The Feerman metric and pseudohermitian invariants," Transactions of the American Mathematical Society, vol. 350, no. 11, pp. 4633-4649, 1998.

[21] E. Barletta, S. Dragomir, and K. L. Duggal, Foliations in CauchyRiemann Geometry, vol. 140 of Mathematical Surveys and Monographs, American Mathematical Society, Providence, RI, USA, 2007.

[22] L. Capogna and N. Garofalo, "Regularity of minimizers of the calculus of variations in Carnot groups via hypoellipticity of systems of Hörmander type," Journal of the European Mathematical Society, vol. 5, no. 1, pp. 1-40, 2003. 
[23] Z.-R. Zhou, "A note on boundary regularity of subelliptic harmonic maps," Kodai Mathematical Journal, vol. 28, no. 3, pp. 525-533, 2005.

[24] C.-J. Xu and C. Zuily, "Higher interior regularity for quasilinear subelliptic systems," Calculus of Variations and Partial Differential Equations, vol. 5, no. 4, pp. 323-343, 1997.

[25] J. Nash, "The embedding problem for Riemannian manifolds," Annals of Mathematics, vol. 63, no. 1, pp. 20-63, 1956.

[26] H. Urakawa, Calculus of Variations and Harmonic Maps, vol. 132 of Translations of Math. Monographs, American Mathematical Society, Providence, RI, USA, 1993.

[27] G. D’Ambra, "Nash C1-embedding theorem for CarnotCaratheodory metrics," Differential Geometry and its Applications, vol. 5, no. 2, pp. 105-119, 1995.

[28] R. S. Strichartz, "Sub-Riemannian geometry," Journal of Differential Geometry, vol. 24, pp. 221-263, 1986.

[29] J. M. Bony, "Principe du maximum, inégalité de Harnack et unicité du problème de Cauchy pour les opérateurs elliptiques dégénérés," Annales de l'institut Fourier, vol. 19, no. 1, pp. 277304, 1969.

[30] F. Hélein, "Regularite des applications faiblement harmoniques entre une surface et une sphere," Comptes Rendus de l'Académie des Sciences, vol. 312, pp. 591-596, 1991.

[31] A. Bensoussan and J. Frehse, Regularity Results for Nonlinear Elliptic Systems and Applications, vol. 151 of Applied Mathematical Sciences, Springer, Berlin, Germany, 2002.

[32] K. O. Widman, "Holder continuity of solutions of elliptic equations," Manuscripta Mathematica, vol. 5, no. 4, pp. 299-308, 1971.

[33] G. B. Folland, "A fundamental solution for a subelliptic operator," Bulletin of the American Mathematical Society, vol. 79, no. 2, pp. 373-376, 1973.

[34] G. Citti, N. Garofalo, and E. Lanconelli, "Harnak's inequality for sum of squares plus potential," American Journal of Mathematics, vol. 115, no. 3, pp. 699-734, 1993.

[35] A. Sánchez-Calle, "Fundamental solutions and geometry of the sum of squares of vector fields," Inventiones Mathematicae, vol. 78, no. 1, pp. 143-160, 1984.

[36] B. Franchi, G. Lu, and R. L. Wheeden, "Representation formulas and weighted Poincaré inequalities for Hörmander vector fields," Annales de l'institut Fourier, vol. 45, no. 2, pp. 577-604, 1995.

[37] P. Hájlasz and P. Koskela, "Sobolev meets Poincaré," Comptes Rendus de l’Académie des Sciences, vol. 320, pp. 1211-1215, 1995. 


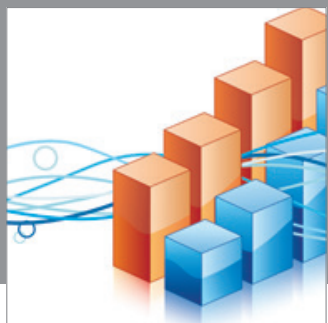

Advances in

Operations Research

mansans

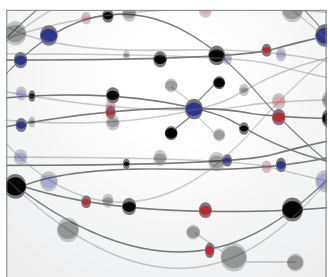

The Scientific World Journal
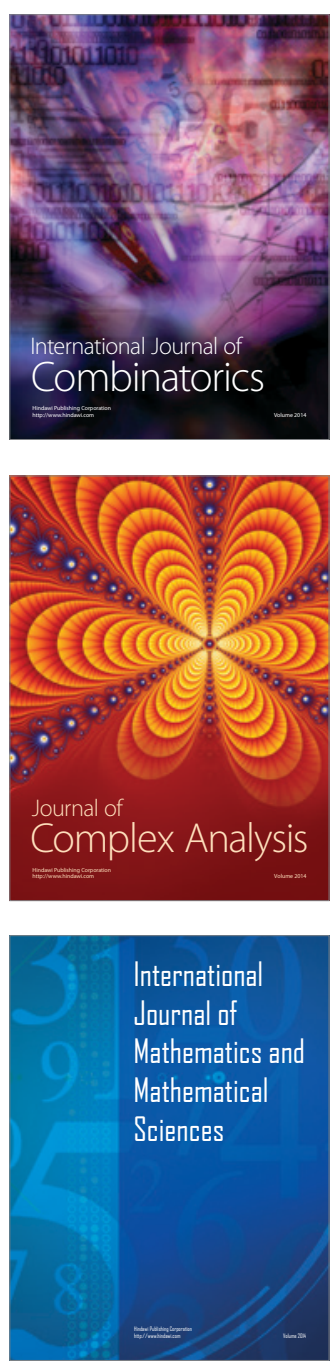
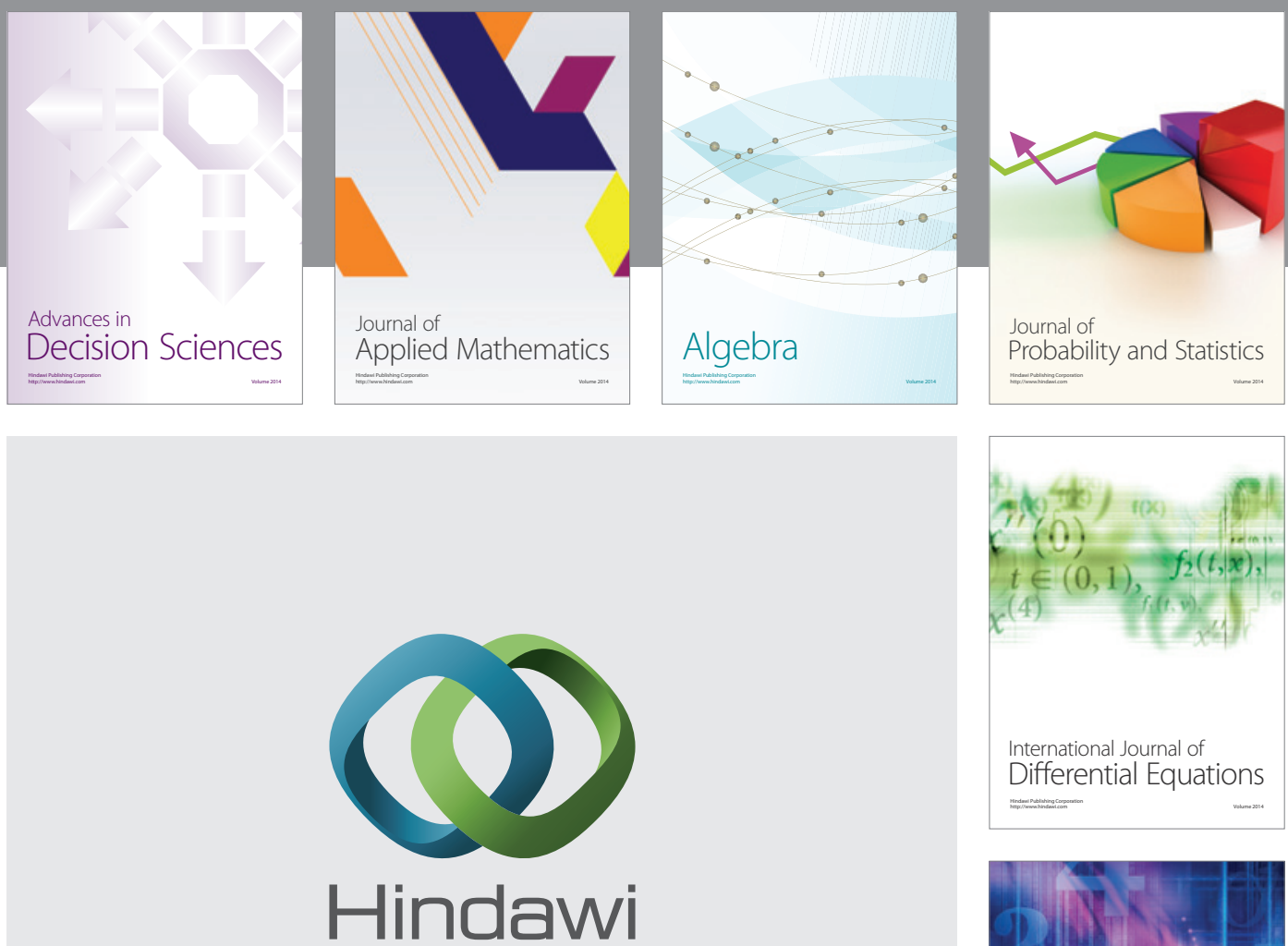

Submit your manuscripts at http://www.hindawi.com
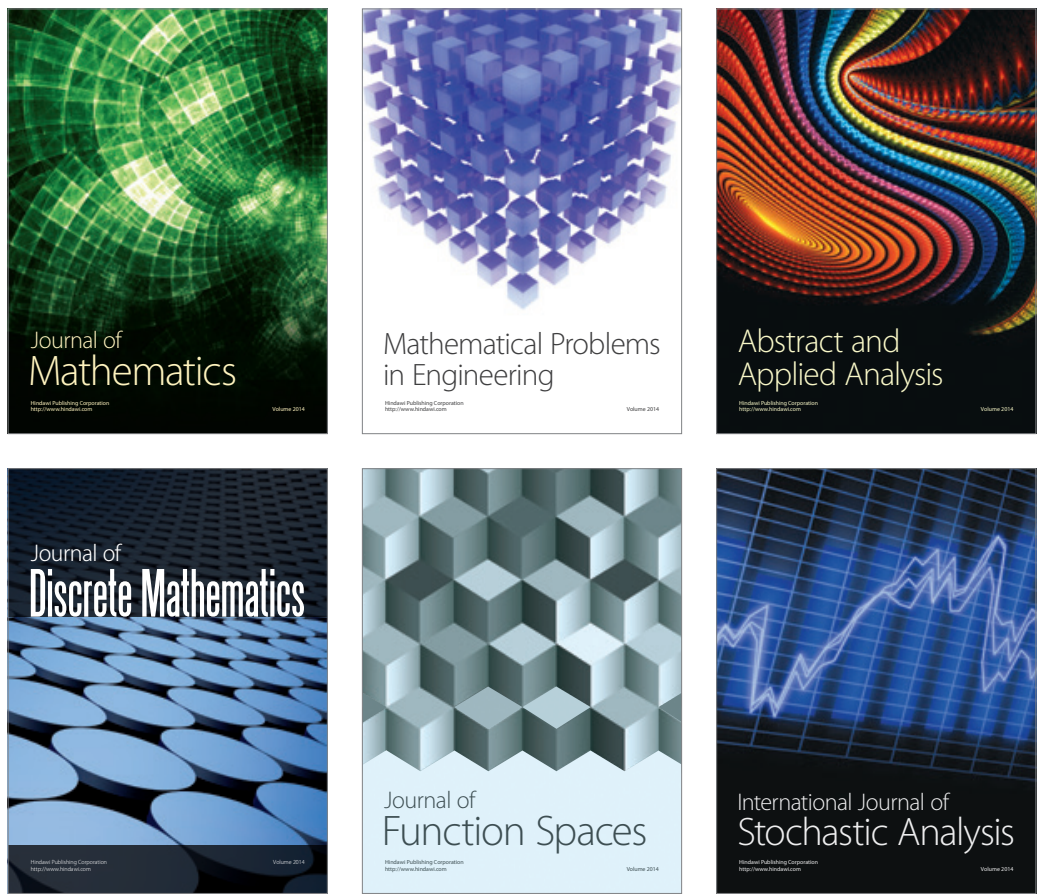

Journal of

Function Spaces

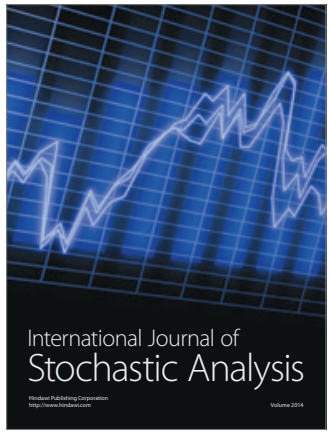

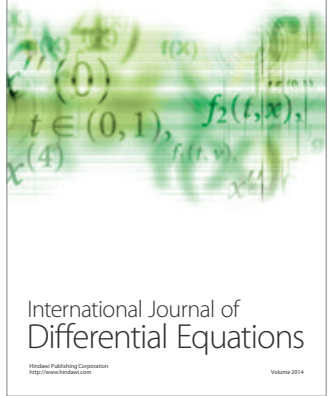
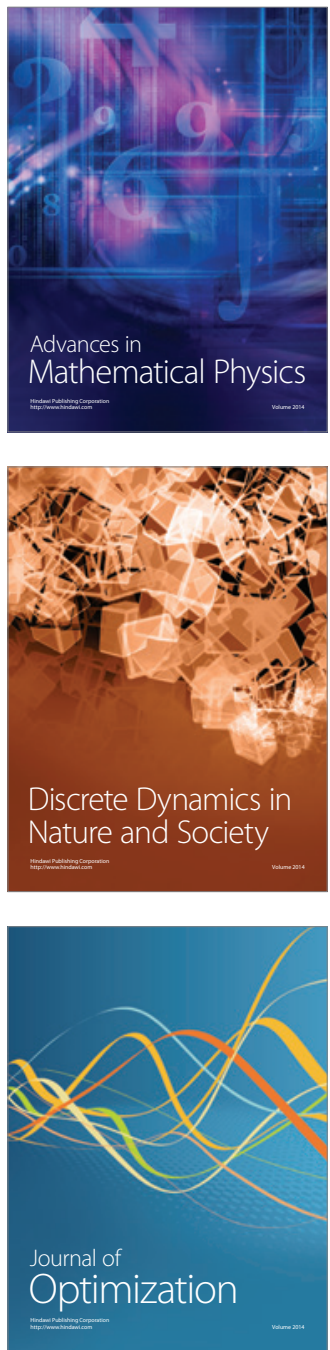